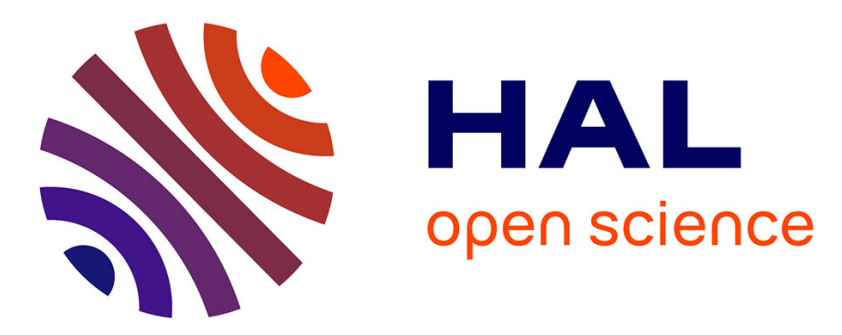

\title{
A switched system approach to exponential stabilization through communication network
}

Alexandre Kruszewski, Wenjuan Jiang, Emilia Fridman, Jean-Pierre Richard, Armand Toguyeni

\section{- To cite this version:}

Alexandre Kruszewski, Wenjuan Jiang, Emilia Fridman, Jean-Pierre Richard, Armand Toguyeni. A switched system approach to exponential stabilization through communication network. IEEE Transactions on Control Systems Technology, 2012, 20 (4), pp.887-900. 10.1109/TCST.2011.2159793 . inria-00602327

\section{HAL Id: inria-00602327 \\ https://hal.inria.fr/inria-00602327}

Submitted on 22 Jun 2011

HAL is a multi-disciplinary open access archive for the deposit and dissemination of scientific research documents, whether they are published or not. The documents may come from teaching and research institutions in France or abroad, or from public or private research centers.
L'archive ouverte pluridisciplinaire HAL, est destinée au dépôt et à la diffusion de documents scientifiques de niveau recherche, publiés ou non, émanant des établissements d'enseignement et de recherche français ou étrangers, des laboratoires publics ou privés. 


\title{
A Switched System Approach to Exponential Stabilization through Communication Network
}

\author{
A. Kruszewski, W.-J. Jiang, E. Fridman, J.-P. Richard, A. Toguyeni
}

\begin{abstract}
The present paper considers a networked control loop, where the plant is a "slave" part, and the remote controller and observer constitute the "master". Since the performance of Networked Control Systems (NCS) depends on the Quality of Service (QoS) available from the network, it is worth to design a controller that takes into account qualitative information on the QoS in realtime. The goal of the design is to provide a controller that guarantees two things: 1) high performances (here expressed by exponential decay rates) when the QoS remains globally the same; 2) global stability when the QoS changes. In order to guarantee the global stability, the controller will switch by respecting a dwell time constraint. The dwell time parameters are obtained by using the switched system theories and the obtained conditions are Linear Matrix Inequalities (LMI). An experiment illustrates how the controller can be implemented for a control over Internet application (remote control of a small robot).
\end{abstract}

\section{Index Terms}

Networked control systems, time delay approach, gain scheduling, Lyapunov-Krasovskii method, LMI

\section{INTRODUCTION}

With the development of computer networks and of communication technologies, real-time control over networks became possible and attracted a lot of attention (see [7], [12], [33], [37] for a general overview on control trends and approaches for networked control systems - NCS).

A. Kruszewski, W.-J. Jiang, J.-P. Richard and A. Toguyeni are with LAGIS CNRS UMR 8146, Ecole Centrale de Lille, BP 48, 59651 Villeneuve d Ascq Cedex, France. J.-P. Richard is also with Equipe-Projet ALIEN, INRIA.

E. Fridman is with School of Electrical Engineering, Tel Aviv University, Tel Aviv 69978, Israel.

E-mail: \{wenjuan.jiang, Alexandre.Kruszewski, jean-pierre.richard, armand.toguyeni\}@ec-lille.fr, emilia@eng.tau.ac.il 
At the same time, in addition to the resulting gain of flexibility, the other expected performances (speed, robustness) also keep growing, and such a demand has to cope with the perturbations the networks induce. Data-packet loss and disorder, time lags depending on the traffic load, asynchronism, bandwidth limitation, belong to such classical drawback of communication networks. This is why real-time control applications classically prefer token ring local area networks, whereas cheaper technologies such as Internet and Ethernet are still limited to less demanding applications such as remote monitoring. The present work aims at both guaranteeing and improving the realtime control performances achievable with classical networks that allow for sending time-stamped packets, including Internet/Ethernet, wireless LAN, Bluetooth, Zigbee, etc.

Many authors already classified the perturbations induced by communication networks and most of them can be regrouped into time-lag effects (see [7], [12], [33], [37] and [19]). Such network-induced delays vary depending on the network hardware, the different protocols, datapacket losses and disorder. This can cause poor performance, instability or danger (see for instance chapter 1 of [27], [8], and the references herein). A variety of stability and control techniques have been developed for general time-delay systems [2], [9], [19], [26]. Many of these techniques consider constant delays. Their adaptation to NCS is either based on simplifying assumptions (considering the time delay as constant [4], [14], [20], [38] is unrealistic in our case, due to the dynamic character of the network), or lead to technical solutions that make the delay become constant: A delay maximizing strategy [3], [16], [23] ("virtual delay", "buffer", or "waiting" strategy) can be carried out so to make the delay become constant and known. However, it is obvious that maximizing the delay up to its largest value decreases the speed performance of the remote system. Concerning time-varying delays, several other results were developed. Among them, [1], [22] considered a communication delay which value is less than the sensor and controller sampling periods. In the Internet case, this constraint leads to increase the sampling periods up to the maximal network delay, which may be constraining for applications with fast dynamics. Predictor-based techniques were also generalized to variable delays [34] but, in the Internet case, the network delays cannot be modelled nor predicted and this lack of knowledge does not allow for concluding. In [36] and [29], Linear Matrix Inequalities (LMI) allow for guaranteeing the stability of the closed-loop systems despite any variation of the communication delays, provided they stay within some interval, say $\left[h_{\min }, h_{\max }\right]$. In [36], the results are based on Lyapunov-Razumikhin Functions for the continuous-time case, which leads 
to stability without additional performance evaluation. In [29] Lyapunov-Krassovskii Functionals (LKF) are applied and exponential stabilization is considered.

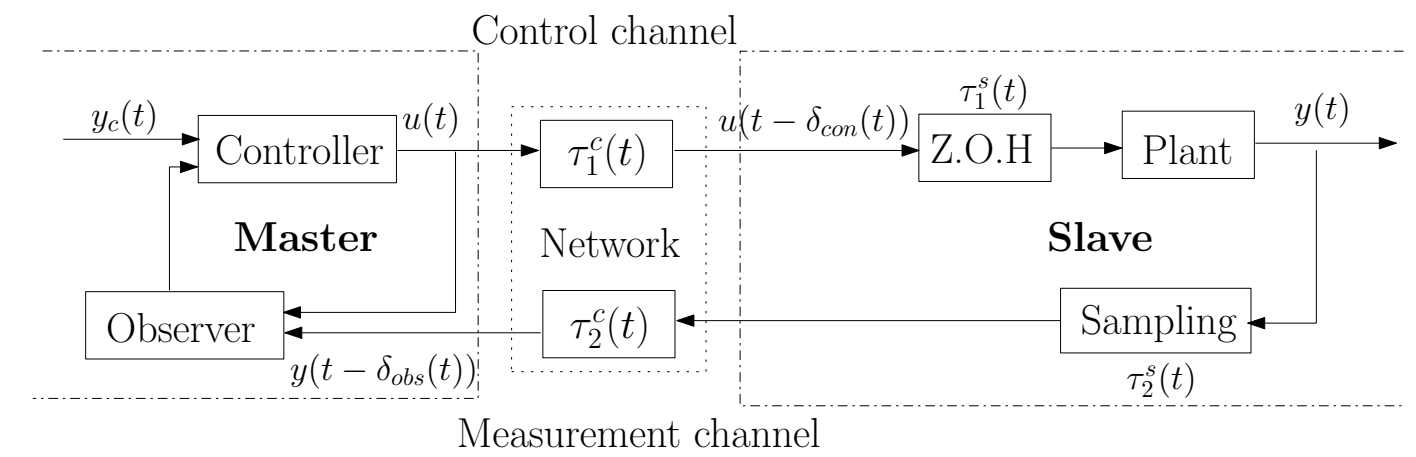

Fig. 1. Feedback NCS with observer-based controller

The structure of NCS that we consider is depicted on Figure 1. It is a single feedback loop which corresponds to the classical simplification of more general NCS, while still capturing many important characteristics of NCS (see [12]). There, a plant (namely, the Slave) is controlled by a remote controller and observer (the Master) via a network. The two communication delays are denoted $\tau_{1}^{c}(t)$ for the control channel (from Master to Slave) and $\tau_{2}^{c}(t)$ for the measurement channel (Slave to Master). Inspired by [29], we limit the waiting delay strategy (aiming at obtaining a constant, virtual delay as recalled above [3], [16], [23]) to the Slave side, while on the side of Master there is no such additional delay. In this way, the measurement channel data can be used directly by the Master as soon as received from the Slave. It will be shown that this structure allows the Master to reconstruct the present Slave's state despite the delays. Note that it could be possible to get rid of the waiting strategy in both the control and measurement channel, and thus to allow a more reactive communication. Such a solution was proposed in [30] at the price of an additional complexity of the LMI involved in the observer design. In the present paper, we prefer to avoid this additional complexity and to focus on the switched gain control, which effects on the performance more essentially.

A main novelty of the present paper lays in the introduction of a QoS-dependent controller structure. In the existing works [7], the stabilization is obtained on the basis of the two bounds $h_{\text {min }}$ and $h_{\text {max }}$, disregarding the way the delays vary between these extrema. If the actual delays stay a long time near to $h_{\text {min }}$, the robust control design keeps taking into account the worst 
case $h_{\max }$ (highest delay, lowest QoS). Such a cautious solution generally yields poor speed performance and we prefer to design a switching controller that adapts to different intervals of delay variation.

In this case, the usual techniques for time-delay systems cannot be directly applied and one has to go to switched systems techniques. Switched systems are dynamical hybrid systems consisting of a family of continuous-time subsystems and a logical rule that orchestrates the switching between them [13], [17]. It is well established that the stability of the subsystems themselves is not sufficient for the stability of the overall system and Lyapunov techniques constitute the basis of the global analysis. In what concerns switched time-delay systems, single [15], [31] and multiple [21] Lyapunov-Krasovskii Functionals (LKFs) methods are the most frequently used for the stabilization of the whole system. A common LKF can ensure the stability with arbitrary switching, but it does not necessarily exist. As an alternative, dwell-time-based switching is considered in [11], [31], [35]. These results on switched time-delay systems consider switched linear systems with the same delays in all the modes.

In this paper, we consider a two-modes switched system with a time-varying delay. We assume that the control input delay $\delta$ may belong to 2 intervals: it is either small (belonging to $\left[h_{1}, h_{2}\right]$ or big (belonging to $\left[h_{2}, h_{3}\right]$ ). Similarly, we assume that the measurement time-varying delay is either small or big. We derive novel conditions for the exponential stability of switched systems with switched delays and coefficients. Next, we solve the output-feedback stabilization problem by designing switched gains for the controller and for the observer. These gains are switched correspondingly to delay switches (such delays are known from the Round-Trip-Time (RTT) measurement and a time-stamp strategy. Of course, more numerous modes could be considered with an increased, off-line computation effort, but this is not to be developed in the present paper.

Finally, differently from the existing works on NCS, we illustrate the efficiency of our theoretical results by experimental ones, where a light-inertia robot is controlled via Internet.

The paper is organized as follows. The features of NCS as well as the problem statement are described in Section II. Section III addresses the exponential stability analysis and the design of switched time-delay systems under arbitrary switching. In Section IV, Multiple LKFs are adapted in the exponential stabilization theory to get a better performance and the global stability is guaranteed by adding the minimum dwell-time into the switched system. The experimental 
example is given in Section $\mathrm{V}$ and confirms the theoretical results.

\section{Problem Statement}

\section{A. Description of the considered system}

In the NCS of Figure 1, the process ("Slave") is a low energy consumption system, the embedded computer of which has a limited computation power. Thus, the control and observation complexity is concentrated on the remote Master computer (the "Master"). This structure makes our theory and application adaptable to sensor and actuator NCSs.

The transmission protocol UDP [24] is applied to communicate the data packets between Master and Slave. It is known that, compared to TCP/IP which retransmits the dropped out data packets, UDP suffers from a lack of reliability. However, in our situation, transmitting fresh data (control data and sampled measurement data) is preferable to retransmitting old ones. Finally, UDP is consuming much less time and is preferred to TCP.

\section{B. The three delay sources}

In Figure 1, the control channel total delay (Master-to-Slave) $\delta_{c o n}(t)$ results from the addition of three (variable) delay sources: 1) The communication through the Internet; 2) The datasampling (see [6]); 3) The possible packet losses (which can be seen as longer sampling in the case of UDP, see [12]). The same phenomenon stands for the measuring channel delay (Slave-to-Master) which total value is denoted $\delta_{o b s}(t)$.

1) Communication: The time-delays of communication $\tau_{i}^{c}(t)$ are variable and not bounded, it is reasonable to assume an upper-bound value since if the time-delay surpasses this value, the packet can be treated as lost.

2) Sampling and packet dropout: The real remote system, including Master, Slave and Network, must involve some data sampling. However, following [6], [28], this phenomenon is equivalent to a time-varying, discontinuous delay. If the sampling period is not negligible, it constitutes a disturbance that should be considered in the stabilization design [36]. If some packet $p_{t_{k}}$ containing the Slave's output (or Master's control data) sampled at $t_{k}$ is lost, or arrives later than the packet $p_{t_{k+1}}$, then the other part only considers the most recent data (i.e., those from $p_{t_{k+1}}$ ). The phenomena acts as a longer sampling period. $N$ is the maximum number of consecutive loss of packet. The packet loss sampling and the sampling induced delay is $\tau_{i}^{S}(t) \leq(N+1) T, i=1,2$. 
In conclusion, the total delays are treated as variable and bounded:

$$
\begin{aligned}
& \delta_{c o n}(t)=\tau_{1}^{c}(t)+\tau_{1}^{s}(t) \\
& \delta_{o b s}(t)=\tau_{2}^{c}(t)+\tau_{2}^{s}(t)
\end{aligned}
$$

\section{Output stabilization}

1) Stabilization for closed-loop system: The master-slaves exchanges are network packets composed by a data and a time-stamp. From the master to slave, the time stamps represent the instant when the master would like to apply the control input. In the other way, the time stamps represent the time $t_{o}$ that the measurement has been done. The clocks of the master and the slave are synchronized before powering up the system. The NTP (Network Time Protocol) [18] is used for this purpose.

The Slave system, is constituted by the plant and a communication system. The communication system is in charge of reordering the packets and applying the control value in the right moment $t_{c}$ given by the master. It also in charge of acquiring the measurement and send them periodically to the master. The measurement time is noted $t_{o}$. by:

$$
\left\{\begin{array}{l}
\dot{x}(t)=A x(t)+B u\left(t_{c}\right), \\
y(t)=C x(t)
\end{array}\right.
$$

where $x(t) \in \mathbb{R}^{n}, u(t) \in \mathbb{R}^{m}, y(t) \in \mathbb{R}^{p}$ are respectively the state, the input and the output vector. $t_{c}$ is the time of the last update of the control value.

The master part is an observer-based state-feedback controller with a communication structure. This latter compares the newest measurements with its respective estimation done at the time $t_{o}$, i.e. when the data was acquired, and send it to the observer structure. The same method is used to send the input value to the observer, ensuring the synchronization of the observer and the plant. This is possible thanks to the buffer in the salve part allowing the master to choose the instant $t_{c}$ that the plant will apply the control value. The delay induced by this buffer strategy must be larger than the maximum delay induced by the communication delay $\tau_{1}^{c}(t)$, i.e. $t_{c}=t+$ buffer $=t+\max \left(\tau_{1}^{c}(t)\right)$. The last task is to send the control values and the corresponding appliance time to the slave. 
The output controller (Master) is based on the following observer:

$$
\left\{\begin{aligned}
\dot{\hat{x}}(t) & =A \hat{x}(t)+B u\left(t_{c}\right)+L\left(y\left(t_{o}\right)-\hat{y}\left(t_{o}\right)\right), \\
\hat{y}(t) & =C \hat{x}(t) \\
u(t) & =K \hat{x}(t)+k y_{c}(t),
\end{aligned}\right.
$$

where $y_{c}(t)$ is the desired setpoint

The estimation error $e(t)=x(t)-\hat{x}(t)$ is given by:

$$
\dot{e}(t)=A e(t)-L C e\left(t_{o}\right) .
$$

Note that the error system does not depend on $u$ nor $x$. This ensures that the separation principle is applicable and allows for reducing the study of the closed-loop dynamic to two smaller stabilization problems. These two problem can be formulated as delay-system stabilization problems [29]:

$$
\begin{aligned}
\text { 1) } \dot{x}(t) & =A x(t)+B K x\left(t-\delta_{c o n}(t)\right) \text {, } \\
\text { 2) } \dot{e}(t) & =A e(t)-L C e\left(t-\delta_{o b s}(t)\right) .
\end{aligned}
$$

where $\delta_{\text {con }}(t)=t-t_{c}, \delta_{\text {con }}(t) \in\left[h_{\min }, h_{\max }\right]$, and $\delta_{o b s}(t)=t-t_{o}, \delta_{o b s}(t) \in\left[h_{\min }, h_{\max },\right]$ being the resulting delays described by .

2) QoS adaptation with switched controller: In order to enhance the performance of the system and take into account the network QoS into the control, the controller switches to the mode $i$ according to the value of the delay. The controller has the information to which predefined subset $S_{i}$ of $\left[h_{\min }, h_{\max }\right]$ belongs the delays. In the following, it is assumed that the two delays $\left\{\delta_{\text {con }}(t), \delta_{o b s}(t)\right\}$ are always in the same subset $S_{i}$ i.e. $\delta_{\text {con }}(t) \in S_{i} \Leftrightarrow \delta_{o b s}(t) \in S_{i}$. The number of modes considered is two i.e. $i \in\{1,2\}$. The two subsets are defined as: $S_{i}=\left[h_{i}, h_{i+1}\right]$ where $\delta(t) \in S_{1}$ represents a small delay, and $\delta(t) \in S_{1}$ represents a big delay. To cover all possible delays, the bounds of the sets are chosen as $h_{1}=h_{\min }$ and $h_{3}=h_{\max }$ then $S_{1} \cap S_{2}=\left[h_{\min }, h_{\max }\right]$.

Remark 1: Only two modes are considered and the same delay subsets are chosen for the controller and the observer for sake of clarity. It is obvious that the results presented here can be extended to more complex cases where the subsets are different for the two delays and where more modes are considered. This is not detailed in this paper due to the heavy burden it may cause to the comprehension. 
The resulting controller differs slightly from the non switching one. In the $i^{\text {th }}$ mode, the time $t_{c}$ of appliance of the control value is based on the maximum of the set $S_{i}, h_{i+1}$. It is given by $t_{c}=t+h_{i+1}-T$. The delay is detected by analyzing the time stamps in the packets, or by measuring the RTT. This introduce a delay in the delay measurement. According to this measure, the switching strategy have the following properties:

1) As soon as a bigger delay is detected the controller must switch to the corresponding mode to minimize disturbances due to a bad size of buffer (buffer < delay).

2) The controller has to follow the dwell time requirements when the detected delay becomes smaller, i.e. in a mode $i$ smaller than the current mode. During the dwell time, the controller artificially enlarge the delay to meet the properties of the current mode.

In its $i^{\text {th }}$ mode, the controller is given by:

$$
\left\{\begin{array}{l}
\dot{\hat{x}}(t)=A \hat{x}(t)+B u\left(t-\hat{\delta}_{c o n}^{i}(t)\right)+L_{i}\left(y\left(t-\delta_{o b s}^{i}(t)\right)-\hat{y}\left(t-\delta_{o b s}^{i}(t)\right)\right), \\
\hat{y}(t)=C \hat{x}(t), \\
u(t)=K_{i} \hat{x}(t)+k y_{c}(t),
\end{array}\right.
$$

where $\hat{\delta}_{\text {con }}^{i}(t)$ is predicted control delay implied by the buffer. $\left.\left.\hat{\delta}_{\text {con }}^{i}(t)\right)=\delta_{\text {con }}^{i}(t)\right)$ when the communication delay is less or equal to the buffer, otherwise $\left.\left.\hat{\delta}_{c o n}^{i}(t)\right) \neq \delta_{c o n}^{i}(t)\right)$ and is not in the same set than $\delta_{c o n}^{i}(t)$. This latter case occurs when the delay moves from the set $S_{1}$ to $S_{2}$, making the buffer smaller that the delay, disturbing the observer (10).

Remark 2: An important feature of this controller is that it has the ability to maintain artificially the delay at an higher value simply by increasing the buffer size. So even if the communication delay is small, we can force $\delta_{c o n}$ to be big, i.e it belongs to $S_{2}$. The global stability will be ensured by a correct use of this ability.

Since a delay is either in $S_{1}$ or in $S_{2}$, one naturally introduces $\chi: \mathbb{R} \rightarrow\{0,1\}$, the characteristic function of $S_{1}$, defined by:

$$
\chi_{s_{1}}(s)=\left\{\begin{array}{l}
1, \text { if } s \in S_{1} \\
0, \text { otherwise. }
\end{array}\right.
$$

The characteristic function of $S_{2}$ is $1-\chi$. Since it is assumed that the two delays are always in the same subsets we have $\chi\left(\delta_{\text {con }}(t)\right)=\chi\left(\delta_{\text {obs }}(t)\right)$. 
Now, considering the control problem (29) together with this 2-modes gain switching strategy, the stabilization problems of observer and controller can be rewritten as follows:

$$
\begin{aligned}
\text { 1) } \dot{x}(t)= & A x(t)+\chi_{S_{1}}\left(\delta_{c o n}(t)\right) B K_{1} \hat{x}\left(t-\delta_{c o n}(t)\right)+\left(1-\chi_{S_{1}}\left(\delta_{c o n}(t)\right)\right) B K_{2} \hat{x}\left(t-\delta_{c o n}(t)\right), \\
\text { 2) } \dot{e}(t)= & A e(t)-\chi_{S_{1}}\left(\delta_{o b s}(t)\right) L_{1} C e\left(t-\delta_{o b s}(t)\right)-\left(1-\chi_{s_{1}}\left(\delta_{o b s}(t)\right)\right) L_{2} C e\left(t-\delta_{o b s}(t)\right) \\
& -B u\left(t-\delta_{c o n}^{i}(t)\right)+B u\left(t-\hat{\delta}_{c o n}^{i}(t)\right) .
\end{aligned}
$$

It must be notified that the separation principle does not hold in this case because of the term $-B u\left(t-\delta_{c o n}^{i}(t)\right)+B u\left(t-\hat{\delta}_{\text {con }}^{i}(t)\right)$. This term equals zero except when a switch from mode 1 to mode 2 occurs. This means that the separation principle holds if there are no switches.

\section{EXPONENTIAL OUTPUT-FEEDBACK STABILIZATION OF SWITCHED TIME-DELAY SYSTEM}

This part provides two results in the general framework of time delay system with switches. The first one gives sufficient conditions for the stability of time delay system with arbitrary switches. The second provides a controller design procedure guaranteeing exponential stability when no switches occurs. These two results are important to design a switching strategy guaranteeing stability and performances.

\section{A. Exponential stability of switched time-delay systems}

Consider the switched system:

$$
\dot{x}(t)=A x(t)+\chi_{S_{1}}(\tau) A_{1} x(t-\tau(t))+\left(1-\chi_{S_{1}}(\tau)\right) A_{2} x(t-\tau(t))
$$

where the delays $\tau \in S_{1} \bigcup S_{2}$ are assumed to be fast-varying (no restrictions on the delayderivative) within these bounds.

The easy way to guarantee the stability of a switched system with arbitrary switches is to find a common LKF for each modes. This has a negative effect on the decay rate $\alpha$ which will be the same in all the modes (the worse one) and it is not the goal of QoS based controller. Another way to deal with switch system is to consider a LKF for each mode and compute some minimum dwell time requirement for stability and performances. This allows performances adaptation according to the current QoS and has a reduced conservatism (in term of finding gains and LKF for some $\alpha$ ). 
For the present problem, the stability analysis is performed by using a pair of LKFs for each modes. In order to be able to compute a dwell time condition, they must be easy to compare so they have the same structure:

$$
\begin{aligned}
& V_{j}\left(t, x_{t}, \dot{x}_{t}\right)=x^{T}(t) P_{j} x(t)+\sum_{i=0}^{2} \int_{t-h_{i+1}}^{t-h_{i}} e^{2 \alpha_{j}(s-t)} x^{T}(s) S_{i j} x(s) d s \\
& +\sum_{i=0}^{2}\left(h_{i+1}-h_{i}\right) \int_{-h_{i+1}}^{-h_{i}} \int_{t+\theta}^{t} e^{2 \alpha_{j}(s-t)} \dot{x}^{T}(s) R_{i j} \dot{x}(s) d s d \theta,
\end{aligned}
$$

where $j$ represents the mode and $h_{0}=0$.

Remark 3: These functionals are taking into account the two possible subsets for the delay. It has no repercussions on the conservatism even in the case where the delay is considered to be in a given subset (for example the delays is considered to belong to $S_{1}$ ). The proof is based on setting the appropriate matrices close to zero.

The following condition along the trajectories of (11)

$$
\dot{V}_{j}\left(t, x_{t}, \dot{x}_{t}\right)+2 \alpha_{j} V_{j}\left(t, x_{t}, \dot{x}_{t}\right) \leq 0
$$

implies some decay rate on the LKF

$$
V_{j}\left(t, x_{t}, \dot{x}_{t}\right) \leq e^{-2 \alpha_{j}\left(t-t_{0}\right)} V_{j}\left(t, x_{t_{0}}, \dot{x}_{t_{0}}\right) \quad \forall t_{0} \in \mathbb{R}
$$

The latter implies exponential stability of (11) since

$$
x^{T}(t) P_{j} x(t) \leq V_{j}\left(t, x_{t}, \dot{x}_{t}\right) \leq e^{-2 \alpha_{j}\left(t-t_{0}\right)} V_{j}\left(t, x_{t_{0}}, \dot{x}_{t_{0}}\right) \leq e^{-2 \alpha_{j}\left(t-t_{0}\right)} V_{j}\left(t, x_{t_{0}}, \dot{x}_{t_{0}}\right)_{\mid \alpha_{j}=0} .
$$

The following theorem gives conditions guaranteeing some decay rate in each mode separately. It DOES NOT ensure stability of the system (11) for arbitrary switches.

Theorem 1: Given $\alpha_{j}>0, j=1,2$, if there exist $n \times n$-matrices $P_{j}>0, R_{i j}>0, S_{i j}>0, i=$ $0,1,2, P_{2 j}, P_{3 j}, Y_{1 j}$ and $Y_{2 j}$ such that the LMIs (15), (16) with (17) are feasible, then each mode $j=1,2$ of switched delay system (11) is exponentially stable with the rate $\alpha_{j}$ for all fast-varying delays $\tau \in\left[h_{j}, h_{j+1}\right]$.

$$
\begin{aligned}
& \left.\Phi\right|_{\chi=1}= \\
& {\left[\begin{array}{ccccccc}
\Phi_{111} & \Phi_{121} & \tilde{R}_{01}+P_{21}^{T} A_{1}-Y_{11}^{T} & Y_{11}^{T} & 0 & {\left[Y_{11}^{T}-P_{21}^{T} A_{1}\right]} & Y_{11}^{T} \\
* & \Phi_{221} & P_{31}^{T} A_{1}-Y_{21}^{T} & Y_{21}^{T} & 0 & {\left[Y_{21}^{T}-P_{31}^{T} A_{1}\right]} & Y_{21}^{T} \\
* & * & \hat{S}_{11}-\left(\tilde{S}_{01}+\tilde{R}_{01}\right) & 0 & 0 & 0 & 0 \\
* & * & * & \hat{S}_{21}-\left(\tilde{S}_{11}+\tilde{R}_{21}\right) & \tilde{R}_{21} & 0 & 0 \\
* & * & * & * & -\left(\tilde{S}_{21}+\tilde{R}_{21}\right) & 0 & 0 \\
* & * & * & * & * & -\tilde{R}_{11} & 0 \\
* & * & * & * & * & * & -\tilde{R}_{11}
\end{array}\right]<0}
\end{aligned}
$$




$$
\begin{aligned}
& \left.\Phi\right|_{\chi=0}= \\
& {\left[\begin{array}{ccccccc}
\Phi_{112} & \Phi_{122} & \tilde{R}_{02} & P_{22}^{T} A_{2}-Y_{12}^{T} & Y_{12}^{T} & {\left[Y_{12}^{T}-P_{22}^{T} A_{2}\right]} & Y_{12}^{T} \\
* & \Phi_{222} & 0 & P_{32}^{T} A_{2}-Y_{22}^{T} & Y_{22}^{T} & {\left[Y_{22}^{T}-P_{32}^{T} A_{2}\right]} & Y_{22}^{T} \\
* & * & \hat{S}_{12}-\left(\tilde{S}_{02}+\tilde{R}_{02}+\tilde{R}_{12}\right) & \tilde{R}_{12} & 0 & 0 & 0 \\
* & * & * & \hat{S}_{22}-\left(\tilde{S}_{12}+\tilde{R}_{12}\right) & 0 & 0 & 0 \\
* & * & * & * & -\tilde{S}_{22} & 0 & 0 \\
* & * & * & * & * & -\tilde{R}_{22} & 0 \\
* & * & * & * & * & * & -\tilde{R}_{22}
\end{array}\right]<0}
\end{aligned}
$$

holds, where

$$
\begin{aligned}
& \tilde{R}_{i j}=e^{-2 \alpha_{j}\left(h_{i+1}\right)} R_{i j}, \\
& \tilde{S}_{i j}=e^{-2 \alpha_{j} h_{i+1}} S_{i j}, \\
& \hat{S}_{i j}=e^{-2 \alpha_{j} h_{i}} S_{i j}, \\
& \Phi_{11 j}=A^{T} P_{2 j}+P_{2 j}^{T} A+S_{0 j}-e^{-2 \alpha_{j} h_{1}} R_{0 j}+2 \alpha_{j} P_{j}, \\
& \Phi_{12 j}=P_{j}-P_{2 j}^{T}+A^{T} P_{3 j}, \Phi_{22 j}=-P_{3 j}-P_{3 j}^{T}+\sum_{i=0}^{2}\left(h_{i+1}-h_{i}\right)^{2} R_{i j} .
\end{aligned}
$$

Proof: Computing (13) with the LKFs (12) gives:

$$
\begin{aligned}
& \dot{V}_{j}\left(t, x_{t}, \dot{x}_{t}\right)+2 \alpha_{j} V_{j}\left(t, x_{t}, \dot{x}_{t}\right) \leq 2 x^{T}(t) P_{j} \dot{x}(t)+2 \alpha_{j} x^{T}(t) P_{j} x(t) \\
& +\dot{x}^{T}(t)\left[\sum_{i=0}^{2}\left(h_{i+1}-h_{i}\right)^{2} R_{i j}\right] \dot{x}(t)-\sum_{i=0}^{2}\left(h_{i+1}-h_{i}\right) e^{-2 \alpha_{j}\left(h_{i+1}\right)} \int_{t-h_{i+1}}^{t-h_{i}} \dot{x}^{T}(s) R_{i j} \dot{x}(s) d s+ \\
& \sum_{i=0}^{2} x\left(t-h_{i}\right)^{T} e^{-2 \alpha_{j} h_{i}} S_{i j} x\left(t-h_{i}\right)-\sum_{i=0}^{2} e^{-2 \alpha_{j} h_{i+1}} x^{T}\left(t-h_{i+1}\right) S_{i j} x\left(t-h_{i+1}\right) .
\end{aligned}
$$

1) We start with the case of $\chi=1$, i.e. $\tau \in\left[h_{1}, h_{2}\right], i=0,2, j=1$. Note that:

$$
\int_{t-h_{2}}^{t-h_{1}} \dot{x}^{T}(s) R_{11} \dot{x}(s) d s=\int_{t-h_{2}}^{t-\tau(t)} \dot{x}^{T}(s) R_{11} \dot{x}(s) d s+\int_{t-\tau(t)}^{t-h_{1}} \dot{x}^{T}(s) R_{11} \dot{x}(s) d s
$$

Applying the Jensen's inequality [9] for $i=0,2$ gives:

$$
\begin{aligned}
& \int_{t-h_{2}}^{t-\tau(t)} \dot{x}^{T}(s) R_{11} \dot{x}(s) d s \geq \frac{1}{h_{2}-h_{1}} \int_{t-h_{2}}^{t-\tau(t)} \dot{x}^{T}(s) d s R_{11} \int_{t-h_{2}}^{t-\tau(t)} \dot{x}(s) d s, \\
& \int_{t-\tau(t)}^{t-h_{1}} \dot{x}^{T}(s) R_{11} \dot{x}(s) d s \geq \frac{1}{h_{2}-h_{1}} \int_{t-\tau(t)}^{t-h_{1}} \dot{x}^{T}(s) d s R_{11} \int_{t-\tau(t)}^{t-h_{1}} \dot{x}(s) d s \\
& \int_{t-h_{i+1}}^{t-h_{i}} \dot{x}^{T}(s) R_{i 1} \dot{x}(s) d s \geq \frac{1}{h_{i+1}-h_{i}} \int_{t-h_{i+1}}^{t-h_{i}} \dot{x}^{T}(s) d s R_{i 1} \int_{t-h_{i+1}}^{t-h_{i}} \dot{x}(s) d s,
\end{aligned}
$$

Then, denoting $v_{11}=\int_{t-\tau(t)}^{t-h_{1}} \dot{x}(s) d s, \quad v_{12}=\int_{t-h_{2}}^{t-\tau(t)} \dot{x}(s) d s$, we obtain:

$$
\begin{aligned}
& \dot{V}_{1}\left(t, x_{t}, \dot{x}_{t}\right)+2 \alpha_{1} V_{1}\left(t, x_{t}, \dot{x}_{t}\right) \leq 2 x^{T}(t) P_{1} \dot{x}(t)+2 \alpha_{1} x^{T}(t) P_{1} x(t) \\
& \left.+\dot{x}^{T}(t) \sum_{i=0}^{2}\left(h_{i+1}-h_{i}\right)^{2} R_{i 1}\right) \dot{x}(t)+\sum_{i=0}^{2} e^{-2 \alpha_{1} h_{i}} x^{T}\left(t-h_{i}\right) S_{i 1} x\left(t-h_{i}\right) \\
& -\sum_{i=0}^{2} e^{-2 \alpha_{1} h_{i+1}} x^{T}\left(t-h_{i+1}\right) S_{i 1} x\left(t-h_{i+1}\right)-\left[x(t)-x\left(t-h_{1}\right)\right]^{T} e^{-2 \alpha_{1} h_{1}} R_{01}\left[x(t)-x\left(t-h_{1}\right)\right] \\
& -\left[x\left(t-h_{2}\right)-x\left(t-h_{3}\right)\right]^{T} e^{-2 \alpha_{1} h_{3}} R_{21}\left[x\left(t-h_{2}\right)-x\left(t-h_{3}\right)\right]-v_{11}^{T} e^{-2 \alpha_{1} h_{2}} R_{11} v_{11}-v_{12}^{T} e^{-2 \alpha_{1} h_{2}} R_{11} v_{12} .
\end{aligned}
$$


We use further the descriptor method [5]. The following quantity:

$$
0=2\left[x^{T}(t) P_{21}^{T}+\dot{x}^{T}(t) P_{31}^{T}\right]\left[A x(t)+A_{1} x\left(t-h_{1}\right)-A_{1} v_{11}-\dot{x}(t)\right],
$$

with some matrices $P_{21}, P_{31}$ of the appropriate size, is added into the right-hand side of (20). Following [10], we also add free weighting matrices:

$$
0=2\left[x^{T}(t) Y_{11}^{T}+\dot{x}^{T}(t) Y_{21}^{T}\right]\left[-x\left(t-h_{1}\right)+x\left(t-h_{2}\right)+v_{11}+v_{12}\right] .
$$

Setting $\eta_{j}(t)=\operatorname{col}\left\{x(t), \dot{x}(t), x\left(t-h_{1}\right), x\left(t-h_{2}\right), x\left(t-h_{3}\right), v_{11}, v_{12}\right\}$, we obtain that the inequality:

$$
\dot{V}_{1}\left(t, x_{t}, \dot{x}_{t}\right)+2 \alpha_{1} V_{1}\left(t, x_{t}, \dot{x}_{t}\right) \leq \eta_{1}^{T}(t) \Phi \eta_{1}(t)<0,
$$

is satisfied if the LMI (15) holds.

2) Continuing with the case $\chi=0$, i.e. $\tau \in\left[h_{2}, h_{3}\right]$, by applying the same method with $j=2$ and $i=0,1$, we obtain:

$$
\begin{aligned}
& \dot{V}_{2}\left(t, x_{t}, \dot{x}_{t}\right)+2 \alpha_{2} V_{2}\left(t, x_{t}, \dot{x}_{t}\right) \leq 2 x^{T}(t) P_{2} \dot{x}(t)+2 \alpha_{2} x^{T}(t) P_{2} x(t) \\
& \left.+\dot{x}^{T}(t) \sum_{i=0}^{2}\left(h_{i+1}-h_{i}\right)^{2} R_{i 2}\right) \dot{x}(t)+\sum_{i=0}^{2} x^{T}\left(t-h_{i}\right) \hat{S}_{i 2} x\left(t-h_{i}\right) \\
& -\sum_{i=0}^{2} x^{T}\left(t-h_{i+1}\right) \tilde{S}_{i 2} x\left(t-h_{i+1}\right)-\left[x(t)-x\left(t-h_{1}\right)\right]^{T} \tilde{R}_{02}\left[x(t)-x\left(t-h_{1}\right)\right] \\
& -\left[x\left(t-h_{2}\right)-x\left(t-h_{1}\right)\right]^{T} \tilde{R}_{21}\left[x\left(t-h_{2}\right)-x\left(t-h_{1}\right)\right]-v_{j 1}^{T} \tilde{R}_{22} v_{j 1}-v_{j 2}^{T} \tilde{R}_{22} v_{j 2} .
\end{aligned}
$$

The equation (21) with $j=2$ is then satisfied if the LMI (16) holds.

This concludes the proof of Theorem 1 .

Remark 4: Note that the Theorem 1 can also be easily applied to arbitrary switches by using the same LKF for both the switching modes, i.e., fixing $\alpha_{1}=\alpha_{2}=\alpha, P_{1}=P_{2}=P, S_{i 1}=S_{i 2}=$ $S_{i}, R_{i 1}=R_{i 2}=R_{i}, P_{21}=P_{22}=P_{2}, P_{31}=P_{32}=P_{3},(i=0,1,2)$.

Form this remark, the following results can be obtained.

Corollary 1: Given $\alpha>0$, if there exist $n \times n$-matrices $P>0, R_{i}>0, S_{i}>0, i=0,1,2, P_{2}$, $P_{3}, Y_{j 1}$ and $Y_{j 2}, j=1,2$ such that the LMIs (15), (16) with notations given in (17) are feasible, then the arbitrary switching delay system (11) is exponentially stable with the rate $\alpha$ for all fast-varying delays $\tau \in\left[h_{j}, h_{j+1}\right], j=1,2$. 


\section{B. Strategy for the output stabilization}

The results presented here are direct consequence of theorem 1. They provide a design procedure to guarantee prescribed performances in each mode. As it was the case for theorem 1 it DOES NOT ensure the stability when arbitrary switches occurs. That is why it is assumed that in (9), $\hat{x}(t)=x(t)$, and in (10), $\hat{\delta}_{c o n}^{i}(t)=\delta_{c o n}^{i}(t)$ (separation principle holds). The first lemma is used for the state feedback design procedure and the second for the observer design procedure.

Lemma 1: Given some scalars $\alpha_{j}>0$ and $\varepsilon_{j}>0, j=1,2$ if there exist some matrices $P_{1 j}>0$, $R_{i j}>0, S_{i j}>0, P_{2 j}, P_{3 j}, Y_{i j}$, and $M_{j}$ such that the LMIs (23) and (24) hold, then each mode $j$ of the closed loop (9) with $\hat{x}(t)=x(t)$ is exponentially stabilized with the rate $\alpha_{j}$. The control gains are given by $K_{j}=M_{j} P_{2 j}^{-1}$.

$$
\begin{aligned}
& \Phi_{\text {con } 1}=\left[\begin{array}{ccccccc}
\Phi_{111} & \Phi_{121} & {\left[\tilde{R}_{01}-B M_{1}-Y_{11}^{T}\right]} & Y_{11}^{T} & {\left[Y_{11}^{T}+B M_{1}\right]} & Y_{11}^{T} & 0 \\
* & \Phi_{221} & {\left[-\varepsilon_{1} B M_{1}-Y_{12}^{T}\right]} & Y_{21}^{T} & {\left[Y_{21}^{T}+\varepsilon_{1} B M_{1}\right]} & Y_{21}^{T} & 0 \\
* & * & \hat{S}_{11}-\tilde{S}_{01}-\tilde{R}_{01} & 0 & 0 & 0 & 0 \\
* & * & * & \hat{S}_{21}-\tilde{S}_{11}-\tilde{R}_{21} & 0 & 0 & \tilde{R}_{21} \\
* & * & * & * & -\tilde{R}_{11} & 0 & 0 \\
* & * & * & * & * & -\tilde{R}_{11} & 0 \\
* & * & * & * & * & * & -\tilde{S}_{21}-\tilde{R}_{21}
\end{array}\right]<0, \\
& \Phi_{\text {con } 2}=\left[\begin{array}{ccccccc}
\Phi_{112} & \Phi_{122} & {\left[-B M_{2}-Y_{12}^{T}\right]} & Y_{12}^{T} & {\left[Y_{12}^{T}+B M_{2}\right]} & Y_{12}^{T} & \tilde{R}_{02} \\
* & \Phi_{222} & {\left[-\varepsilon_{2} B M_{2}-Y_{22}^{T}\right]} & Y_{22}^{T} & {\left[Y_{22}^{T}+\varepsilon_{2} B M_{2}\right]} & Y_{22}^{T} & 0 \\
* & * & \hat{S}_{22}-\tilde{S}_{12}-\tilde{R}_{12} & 0 & 0 & 0 & \tilde{R}_{12} \\
* & * & * & -\tilde{S}_{22} & 0 & 0 & 0 \\
* & * & * & * & -\tilde{R}_{22} & 0 & 0 \\
* & * & * & * & * & -\tilde{R}_{22} & 0 \\
* & * & * & * & * & * & \hat{S}_{12}-\tilde{S}_{02}-\tilde{R}_{02}-\tilde{R}_{12}
\end{array}\right]<0,
\end{aligned}
$$

with:

$$
\begin{aligned}
& \tilde{R}_{i j}=e^{-2 \alpha_{j} h_{i+1}} R_{i j}, \\
& \tilde{S}_{i j}=e^{-2 \alpha_{j} h_{i+1}} S_{i j}, \\
& \hat{S}_{i j}=e^{-2 \alpha_{j} h_{i}} S_{i j}, \\
& \Phi_{11 j}=A P_{2 j}+P_{2 j}^{T} A^{T}+S_{0 j}-e^{-2 \alpha_{j} h_{1}} R_{0 j}+2 \alpha_{j} P_{1 j}, \\
& \Phi_{12 j}=P_{1 j}-P_{2 j}+\varepsilon_{j} P_{2 j}^{T} A, \\
& \Phi_{22 j}=-\varepsilon_{j} P_{2 j}-\varepsilon_{j} P_{2 j}^{T}+\sum_{i=0}^{2}\left(h_{i+1}-h_{i}\right)^{2} R_{i j} .
\end{aligned}
$$

Lemma 2: Given some scalars $\alpha_{j}>0$ and $\varepsilon_{j}>0, j=1,2$, if there exist some matrices $P_{1 j}>0$, $R_{i j}>0, S_{i j}>0, P_{2 j}, P_{3 j}, Y_{i j}$, and $M_{j}$ such that the following LMIs (26) and (27) hold, then each mode of the observation error equation given in (10) with $\hat{\delta}_{c o n}^{i}(t)=\delta_{c o n}^{i}(t)$ is exponentially 
stabilized with a decay rate $\alpha_{j}$ :

$$
\begin{aligned}
& \Phi_{o b s 1}=\left[\begin{array}{ccccccc}
\Phi_{111} & \Phi_{121} & {\left[\tilde{R}_{01}-W_{1} C-Y_{11}^{T}\right]} & Y_{11}^{T} & {\left[Y_{11}^{T}+W_{1} C\right]} & Y_{11}^{T} & 0 \\
* & \Phi_{221} & {\left[-\varepsilon_{1} W_{1} C-Y_{21}^{T}\right]} & Y_{21}^{T} & {\left[Y_{21}^{T}+\varepsilon_{1} W_{1} C\right]} & Y_{21}^{T} & 0 \\
* & * & \hat{S}_{11}-\tilde{S}_{01}-\tilde{R}_{01} & 0 & 0 & 0 & 0 \\
* & * & * & \hat{S}_{21}-\tilde{S}_{11}-\tilde{R}_{21} & 0 & 0 & \tilde{R}_{21} \\
* & * & * & * & -\tilde{R}_{11} & 0 & 0 \\
* & * & * & * & * & -\tilde{R}_{11} & 0 \\
* & * & * & * & * & * & -\tilde{S}_{21}-\tilde{R}_{21}
\end{array}\right]<0, \\
& \Phi_{\text {obs } 2}=\left[\begin{array}{ccccccc}
\Phi_{112} & \Phi_{122} & {\left[-W_{2} C-Y_{12}^{T}\right]} & Y_{12}^{T} & {\left[Y_{12}^{T}+W_{2} C\right]} & Y_{12}^{T} & \tilde{R}_{02} \\
* & \Phi_{222} & {\left[-\varepsilon_{2} W_{2} C-Y_{22}^{T}\right]} & Y_{22}^{T} & {\left[Y_{22}^{T}+\varepsilon_{2} W_{2} C\right]} & Y_{22}^{T} & 0 \\
* & * & \hat{S}_{22}-\tilde{S}_{12}-\tilde{R}_{12} & 0 & 0 & 0 & \tilde{R}_{12} \\
* & * & * & -\tilde{S}_{22} & 0 & 0 & 0 \\
* & * & * & * & -\tilde{R}_{22} & 0 & 0 \\
* & * & * & * & * & -\tilde{R}_{22} & 0 \\
* & * & * & * & * & * & \hat{S}_{12}-\tilde{S}_{02}-\tilde{R}_{02}-\tilde{R}_{12}
\end{array}\right]<0,
\end{aligned}
$$

with:

$$
\begin{aligned}
& \tilde{R}_{i j}=e^{-2 \alpha_{j} h_{i+1}} R_{i j}, \\
& \tilde{S}_{i j}=e^{-2 \alpha_{j} h_{i+1}} S_{i j}, \\
& \hat{S}_{i j}=e^{-2 \alpha_{j} h_{i}} S_{i j}, \\
& \Phi_{11 j}=A^{T} P_{2 j}+P_{2 j}^{T} A+S_{0 j}-e^{-2 \alpha h_{1}} R_{0 j}+2 \alpha_{j} P_{1 j}, \\
& \Phi_{12 j}=P_{1 j}-P_{2 j}^{T}+\varepsilon_{j} A^{T} P_{2 j}, \\
& \Phi_{22 j}=-\varepsilon P_{2 j}-\varepsilon P_{2 j}^{T}+\sum_{i=0}^{2}\left(h_{i+1}-h_{i}\right)^{2} R_{i j} .
\end{aligned}
$$

Proof: Both proofs remain the same as for theorem 1. Only some matrix matrix manipulation and changes of variables are added. For more details, similar proof are in [15], [32].

\section{GLOBAL STABILIZATION OF THE NETWORKED CONTROL SYSTEM WITH MINIMUM DWELL TIME}

The previous part was devoted to design the state feedback and the observer to ensure some decay rate while the system does not switch. The present part gives material to design the switching rule of the controller guaranteeing stability with/o performances. The main idea is to exploit the ability of the controller to enlarge the delay by using buffer and avoid some switching occurrences. This feature permit to respect some dwell time requirement for the global stability with switches. 


\section{A. Networked control system Model}

This subsection gives the global model of the networked controlled system. This model takes into account the interactions between the delay detection problem, the switching buffer size and the observer. In this part, there are no separation principle and the overall models has to be taken into account. It assumed that the controller gains $K_{i}$ and $L_{i}$ are designed using lemmas 1 and 2 on the non switching model for decay rates $\alpha_{i}$.

Let recall our networked control system.

$$
\left\{\begin{array}{l}
\dot{x}(t)=A x(t)+B u\left(t-\delta_{c o n}(t)\right), \\
y(t)=C x(t)
\end{array}\right.
$$

With its controller:

$$
\left\{\begin{array}{l}
\dot{\hat{x}}(t)=A \hat{x}(t)+B u\left(t-\hat{\delta}_{c o n}^{i}(t)\right)+L_{i}\left(y\left(t-\delta_{o b s}^{i}(t)\right)-\hat{y}\left(t-\delta_{o b s}^{i}(t)\right)\right), \\
\hat{y}(t)=C \hat{x}(t) \\
u(t)=K_{i} \hat{x}(t)+k y_{c}(t),
\end{array}\right.
$$

Remind that the controller switches as soon as a bigger delay is detected but one can choose when it switch if the delay becomes smaller by increasing artificially the delay. The delay is measured with some lag (takes at maximum $h_{\max }$ if measured on the measurement channel).Because of this lag and because of the buffer size problem, four modes arise including two additional modes when the estimated delay does not equals the real one:

$S S_{1}$ mode, $t \in\left[t_{0}, t_{1}\right]$ : The delays are in $S_{1}$ and are correctly detected. In this case, the small buffer is used and the controller is in the mode $i=1$. In that case, $\hat{\delta}_{c o n}(t)=\delta_{c o n}$. The controller gains, $K_{1}$ and $L_{1}$, are designed for exponential stability with a decay rate $\alpha_{1}$. The equations of $S S_{1}$ with $\zeta(t)=\operatorname{col}\{x(t), e(t)\}$ are given by:

$$
\begin{aligned}
& \dot{\zeta}(t)=\bar{A}_{0} \zeta(t)+\bar{A}_{11} \zeta\left(t-\delta_{c o n}^{1}(t)\right)+\bar{A}_{31} \zeta\left(t-\delta_{o b s}^{1}(t)\right) \\
& \delta_{c o n}^{1}(t), \delta_{o b s}^{1}(t) \in\left[h_{1}, h_{2}\right]
\end{aligned}
$$

with $\bar{A}_{0}=\left[\begin{array}{cc}A & 0 \\ 0 & A\end{array}\right], \bar{A}_{11}=\left[\begin{array}{cc}B K_{1} & -B K_{1} \\ 0 & 0\end{array}\right], \bar{A}_{31}=\left[\begin{array}{cc}0 & 0 \\ 0 & -L_{1} C\end{array}\right]$.

$S U_{1}$ mode, $t \in\left[t_{1}, t_{2}\right]$ : The system was in $S S_{1}$ then the delay grows and belongs now in $S_{2}$. The value is not detected yet by the communication structure implying that the controller stays in mode 1. This mode may be unstable since the controller gains $K_{1}$ and $L_{1}$ are not designed for. Its decay rate is denoted by $\alpha_{3}$ and may be negative. The second effect is that the control 
buffer is smaller than the communication delay, the observed control input and the plant control input are no more synchronized during this period because $\hat{\delta}_{\text {con }}(t) \neq \delta_{\text {con }}(t)$. This mode has a maximum dwell time since the delay detection takes less than the maximum communication delay. The equation of $S U_{1}$ are:

$$
\begin{aligned}
& \dot{\zeta}(t)=\bar{A}_{0} \zeta(t)+\bar{A}_{13} \zeta\left(t-\hat{\delta}_{c o n}^{1}(t)\right)+\bar{A}_{23} \zeta\left(t-\delta_{c o n}^{2}(t)\right)+\bar{A}_{33} \zeta\left(t-\delta_{o b s}^{2}(t)\right) \\
& \hat{\delta}_{c o n}^{1} \in\left[h_{1}, h_{2}\right], \delta_{c o n}^{2}(t), \delta_{o b s}^{2}(t) \in\left[h_{2}, h_{3}\right]
\end{aligned}
$$

for $\bar{A}_{0}=\left[\begin{array}{cc}A & 0 \\ 0 & A\end{array}\right], \bar{A}_{13}=\left[\begin{array}{cc}0 & 0 \\ -B K_{1} & B K_{1}\end{array}\right], \bar{A}_{23}=\left[\begin{array}{cc}B K_{1} & -B K_{1} \\ B K_{1} & -B K_{1}\end{array}\right], \bar{A}_{33}=\left[\begin{array}{cc}0 & 0 \\ 0 & -L_{1} C\end{array}\right]$.

$S S_{2}$ mode, $t \in\left[t_{3}, t_{4}\right]$ : The system was in mode $S U_{1}$ and finally detect the correct value of the delay. The controller switches to the mode 2. In that case, the buffer works again properly, i.e. $\hat{\delta}_{\text {con }}(t)=\delta_{\text {con }}(t)$. The controller gains, $K_{2}$ and $L_{2}$, are designed for exponential stability with a decay rate $\alpha_{2}$. The equations are:

$$
\begin{aligned}
& \dot{\zeta}(t)=\bar{A}_{0} \zeta(t)+\bar{A}_{12} \zeta\left(t-\delta_{c o n}^{2}(t)\right)+\bar{A}_{32} \zeta\left(t-\delta_{o b s}^{2}(t)\right) \\
& \delta_{c o n}^{2}(t), \delta_{o b s}^{2}(t) \in\left[h_{2}, h_{3}\right]
\end{aligned}
$$

with $\bar{A}_{0}=\left[\begin{array}{ll}A & 0 \\ 0 & A\end{array}\right], \bar{A}_{12}=\left[\begin{array}{cc}B K_{2} & -B K_{2} \\ 0 & 0\end{array}\right], \bar{A}_{32}=\left[\begin{array}{cc}0 & 0 \\ 0 & -L_{2} C\end{array}\right]$.

$S U_{2}$ mode, $t \in\left[t_{3}, t_{s}\right]$ : The delay becomes smaller and belongs to $S_{1}$ but the controller do not switch to mode $i=1$. It waits to meet the dwell time condition. The control gains $K_{2}$ are still design for this mode since the delay is artificially kept in $S_{2}$. And the observer gains $L_{2}$ keep the stability since if the conditions of theorem 1 hold for a given delay, it does also for a smaller one. The global decay rate is noted $\alpha_{4}$.

$$
\begin{aligned}
& \dot{\zeta}(t)=\bar{A}_{0} \zeta(t)+\bar{A}_{14} \zeta\left(t-\delta_{c o n}^{2}(t)\right)+\bar{A}_{34} \zeta\left(t-\delta_{o b s}^{1}(t)\right) . \\
& \delta_{c o n}^{2}(t) \in\left[h_{2}, h_{3}\right], \delta_{o b s}^{1}(t) \in\left[h_{1}, h_{2}\right]
\end{aligned}
$$

$$
\text { for } \bar{A}_{0}=\left[\begin{array}{ll}
A & 0 \\
0 & A
\end{array}\right], \bar{A}_{14}=\left[\begin{array}{cc}
B K_{2} & -B K_{2} \\
0 & 0
\end{array}\right], \bar{A}_{34}=\left[\begin{array}{cc}
0 & 0 \\
0 & -L_{2} C
\end{array}\right] \text {. }
$$

The four modes is summarized in the following figure where the arrows represent the possible transitions, the only controller transition is $S U 2 \rightarrow S S 1$ : 


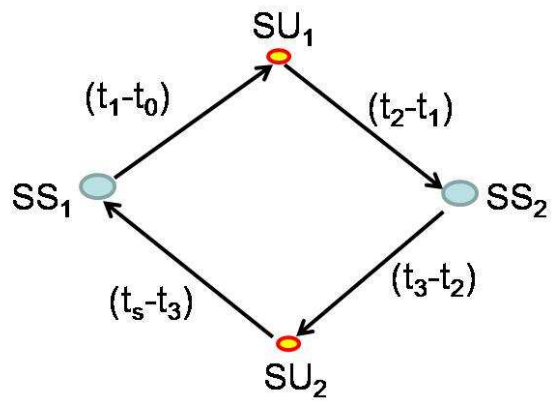

Fig. 2. Switch among four modes.

To summarize:

$$
\begin{aligned}
& S S_{1} \quad: \quad t \in\left[t_{0}, t_{1}\right] \quad i=1, \quad \text { Buffer }=h_{2}-T \quad \hat{\delta}_{\text {con }}(t)=\delta_{\text {con }}(t) \quad \delta_{\text {con }}(t) \in S_{1}, \quad \delta_{\text {obs }}(t) \in S_{1} \\
& S U_{1} \quad: \quad t \in\left[t_{1}, t_{2}\right] \quad i=1, \quad B u f f e r=h_{2}-T \quad \hat{\delta}_{\text {con }}(t) \neq \delta_{\text {con }}(t) \quad \delta_{\text {con }}(t) \in S_{2}, \quad \delta_{\text {obs }}(t) \in S_{2} \\
& S S_{2} \quad: \quad t \in\left[t_{2}, t_{3}\right] \quad i=2, \quad \text { Buffer }=h_{3}-T \quad \hat{\delta}_{\text {con }}(t)=\delta_{\text {con }}(t) \quad \delta_{\text {con }}(t) \in S_{2}, \quad \delta_{\text {obs }}(t) \in S_{2} \\
& S U_{2}: t \in\left[t_{3}, t_{4}\right] \quad i=2, \quad \text { Buffer }=h_{3}-T \quad \hat{\delta}_{\text {con }}(t)=\delta_{\text {con }}(t) \quad \delta_{\text {con }}(t) \in S_{2}, \quad \delta_{\text {obs }}(t) \in S_{1}
\end{aligned}
$$

\section{B. Global stability conditions}

In order to avoid unstable switching sequences, a minimum dwell time between the modes is computed here by using the exponential stability property. The global stability of the system will be studied by using the method of [17]. The LKF $V_{1}, V_{2}, V_{3}$ and $V_{4}$ are respectively associated to the modes $S S_{1}, S S_{2}, S U_{1}$ and $S U_{2}$. A common structure is chosen:

$$
\begin{aligned}
& V_{j}\left(t, x_{t}, \dot{x}_{t}\right)=x^{T}(t) P_{j} x(t)+\sum_{i=0}^{2} \int_{t-h_{i+1}}^{t-h_{i}} e^{2 \alpha_{j}(s-t)} x^{T}(s) S_{i j} x(s) d s \\
& +\sum_{k=1}^{3} \sum_{i=0}^{2}\left(h_{i+1}-h_{i}\right) \int_{-h_{i+1}}^{-h_{i}} \int_{t+\theta}^{t} e^{2 \alpha_{j}(s-t)} \dot{x}^{T}(s) R_{i j}^{k} \dot{x}(s) d s d \theta,
\end{aligned}
$$

LKF are used to compute the decay rate $\alpha_{j}$ of each modes and a scale parameter $\mu_{i j}$ between the modes:

$$
\begin{aligned}
& V_{j}\left(t, x_{t}, \dot{x}_{t}\right) \leq \mu_{i j} V_{i}\left(t, x_{t}, \dot{x}_{t}\right) \forall i, j=1, \ldots, 4 \\
& V_{j}\left(t, x_{t}, \dot{x}_{t}\right) \leq e^{-2 \alpha_{j}\left(t-t_{0}\right)} V_{j}\left(t_{0}, x_{t_{0}}, \dot{x}_{t_{0}}\right) \forall j=1, \ldots, 4
\end{aligned}
$$

The following lemma provides conditions ensuring $V_{j}\left(t, x_{t}, \dot{x}_{t}\right) \leq \mu_{i j} V_{i}\left(t, x_{t}, \dot{x}_{t}\right)$ for the considered sequence. 


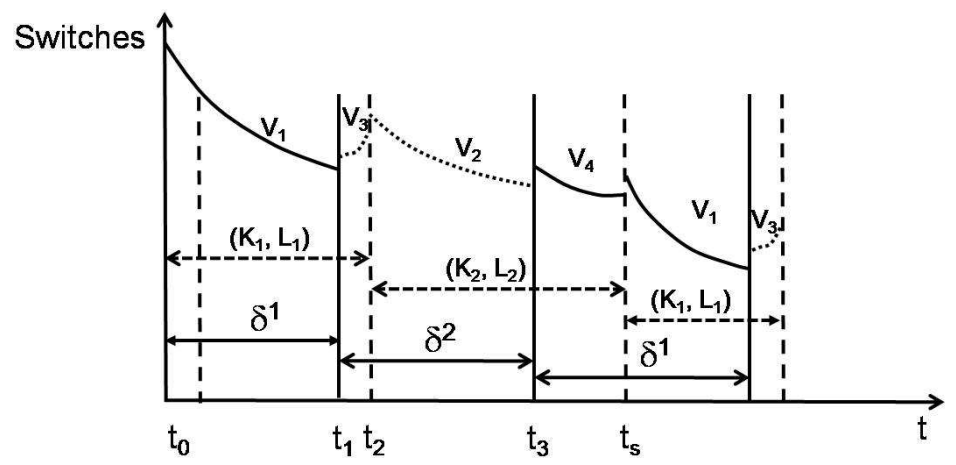

Fig. 3. Minimum dwell time: Small delay from $t_{0}$ to $t_{1}$, then big delay from $t 1$ to $t_{3}$, switches at $t_{2}$ and $t_{s}$.

Lemma 3: The property $\left.V_{j}\left(t, x_{t}, \dot{x}_{t}\right) \leq \mu_{i j} V_{i}\left(t, x_{t}, \dot{x}_{t}\right), \quad(i, j) \in\{(1,4),(4,2),(2,3),(3,1)\}\right\}$ is guaranteed if the following conditions are satisfied :

$$
\begin{aligned}
& \forall n \in\{0,1,2\}, \forall h \in\left\{h_{n}, h_{n+1}\right\}, \forall k \in\{1,2,3\} \\
& P_{i} \leq \mu_{i j} P_{j} \\
& \forall(i, j) \in\{(1,4),(4,2),(2,3),(3,1)\}, \\
& e^{-2 \alpha_{i} h} S_{n i} \leq \mu_{i j} e^{-2 \alpha_{j} h} S_{n j}, \\
& e^{-2 \alpha_{i} h} R_{n i}^{k} \leq \mu_{i j} e^{-2 \alpha_{j} h} R_{n j}^{k} .
\end{aligned}
$$

The computation of the parameters $\alpha_{i}$ and $\mu_{i j}$ is done using the following lemma which is a specific version of theorem 1 .

Lemma 4: Given some scalars $\alpha_{j}$ and $\mu_{i j},(i, j) \in\{(1,4),(4,2),(2,3),(3,1)\}$, if there exist scalars $\mu_{i j}, i, j=1, \ldots, 4$, matrices $P_{j}>0, R_{i j}^{k}>0$ and $S_{i j}>0(i=0,1,2$ and $k=1,2,3), P_{2 j}, P_{3 j}$, $Y_{1 k j}$ and $Y_{2 k j}(j=1,2,3,4$ and $k=1,2,3)$ with proper dimensions, such that (38), (39) and (37) conditions hold then the system (IV-A) has positive functionals (35) fulfilling 36.

$$
\begin{aligned}
& \left.\Phi\right|_{S S_{1}}=\left[\begin{array}{cc}
\Psi_{11} & \Psi_{21} \\
* & \Psi_{31}
\end{array}\right]<0,\left.\quad \Phi\right|_{S S_{2}}=\left[\begin{array}{cc}
\Psi_{12} & \Psi_{22} \\
* & \Psi_{32}
\end{array}\right]<0, \\
& \left.\Phi\right|_{S U_{1}}=\left[\begin{array}{cc}
\Psi_{13} & \Psi_{23} \\
* & \Psi_{33}
\end{array}\right]<0,\left.\quad \Phi\right|_{S U_{2}}=\left[\begin{array}{cc}
\Psi_{14} & \Psi_{24} \\
* & \Psi_{34}
\end{array}\right]<0,
\end{aligned}
$$


where:

$$
\begin{aligned}
& \Psi_{11}=\left[\begin{array}{ccccc}
\Phi_{111} & \Phi_{121} & P_{21}^{T} A_{11}+P_{21}^{T} A_{31}+\sum_{k=1}^{3}\left(\tilde{R}_{01}^{k}-Y_{1 k 1}^{T}\right) & \sum_{k=1}^{3} Y_{1 k 1}^{T} & 0 \\
* & \Phi_{221} & P_{31}^{T} A_{11}+P_{31}^{T} A_{31}-\sum_{k=1}^{3} Y_{2 k 1}^{T} & \sum_{k=1}^{3} Y_{2 k 1}^{T} & 0 \\
* & * & \hat{S}_{11}-\tilde{S}_{01}-\sum_{k=1}^{3} \tilde{R}_{01}^{k} & 0 & 0 \\
* & * & * & \hat{S}_{21}-\tilde{S}_{11}-\sum_{k=1}^{3} \tilde{R}_{21}^{k} & \sum_{k=1}^{3} \tilde{R}_{21}^{k} \\
* & * & * & * & -\tilde{S}_{21}-\sum_{k=1}^{3} \tilde{R}_{21}^{k}
\end{array}\right], \\
& \Psi_{21}=\left[\begin{array}{cccccc}
Y_{11}^{T}-P_{21}^{T} A_{11} & Y_{111}^{T} & Y_{121}^{T} & Y_{121}^{T} & Y_{131}^{T}-P_{21}^{T} A_{31} & Y_{131}^{T} \\
Y_{211}^{T}-P_{31}^{T} A_{11} & Y_{211}^{T} & Y_{221}^{T} & Y_{221}^{T} & Y_{231}^{T}-P_{31}^{T} A_{31} & Y_{231}^{T} \\
0 & 0 & 0 & 0 & 0 & 0 \\
0 & 0 & 0 & 0 & 0 & 0 \\
0 & 0 & 0 & 0 & 0 & 0
\end{array}\right] \\
& \Psi_{31}=\left[\begin{array}{cccccc}
-\tilde{R}_{11}^{1} & 0 & 0 & 0 & 0 & 0 \\
* & -\tilde{R}_{11}^{1} & 0 & 0 & 0 & 0 \\
* & * & -\tilde{R}_{11}^{2} & 0 & 0 & 0 \\
* & * & * & -\tilde{R}_{11}^{2} & 0 & 0 \\
* & * & * & * & -\tilde{R}_{11}^{3} & 0 \\
* & * & * & * & * & -\tilde{R}_{11}^{3}
\end{array}\right] \text {, } \\
& \Psi_{12}=\left[\begin{array}{ccccc}
\Phi_{112} & \Phi_{122} & \sum_{k=1}^{3} \tilde{R}_{02}^{k} & P_{22}^{T} A_{12}+P_{21}^{T} A_{31}-\sum_{k=1}^{3} Y_{1 k 2}^{T} & \sum_{k=1}^{3} Y_{1 k 2}^{T} \\
* & \Phi_{222} & 0 & P_{32}^{T} A_{12}+P_{32}^{T} A_{32}-\sum_{k=1}^{3}\left(Y_{2 k 2}^{T}\right) & \sum_{k=1}^{3} Y_{2 k 2}^{T} \\
* & * & \hat{S}_{12}-\tilde{S}_{02}-\sum_{k=1}^{3}\left(\tilde{R}_{02}^{k}+\tilde{R}_{12}^{k}\right) & \sum_{k=1}^{3} \tilde{R}_{12}^{k} & 0 \\
* & * & * & \hat{S}_{22}-\tilde{S}_{12}-\sum_{k=1}^{3} \tilde{R}_{12}^{k} & 0 \\
* & * & * & * & -\tilde{S}_{22}
\end{array}\right] \text {, } \\
& \Psi_{22}=\left[\begin{array}{cccccc}
Y_{112}^{T}-P_{22}^{T} A_{12} & Y_{112}^{T} & Y_{122}^{T} & Y_{122}^{T} & Y_{132}^{T}-P_{22}^{T} A_{32} & Y_{132}^{T} \\
Y_{212}^{T}-P_{32}^{T} A_{12} & Y_{212}^{T} & Y_{222}^{T} & Y_{222}^{T} & Y_{232}^{T}-P_{32}^{T} A_{32} & Y_{232}^{T} \\
0 & 0 & 0 & 0 & 0 & 0 \\
0 & 0 & 0 & 0 & 0 & 0 \\
0 & 0 & 0 & 0 & 0 & 0
\end{array}\right] \\
& \Psi_{32}=\left[\begin{array}{cccccc}
-\tilde{R}_{22}^{1} & 0 & 0 & 0 & 0 & 0 \\
* & -\tilde{R}_{22}^{1} & 0 & 0 & 0 & 0 \\
* & * & -\tilde{R}_{22}^{2} & 0 & 0 & 0 \\
* & * & * & -\tilde{R}_{22}^{2} & 0 & 0 \\
* & * & * & * & -\tilde{R}_{22}^{3} & 0 \\
* & * & * & * & * & -\tilde{R}_{22}^{3}
\end{array}\right] \text {, }
\end{aligned}
$$$$
\Psi_{13}=\left[\begin{array}{cccccc}
\Phi_{113} & \Phi_{123} & \sum_{k=1}^{3} \tilde{R}_{03}^{k}+P_{23}^{T} \bar{A}_{13}-Y_{113}^{T} & P_{23}^{T}\left(\bar{A}_{23}+\bar{A}_{33}\right)+Y_{113}^{T}-Y_{123}^{T}-Y_{133}^{T} & Y_{123}^{T}+Y_{133}^{T} & \\
* & \Phi_{223} & P_{33}^{T} \bar{A}_{13}-Y_{213}^{T} & P_{33}^{T} \bar{A}_{23}+P_{33}^{T} \bar{A}_{33}+Y_{213}^{T}-Y_{223}^{T}-Y_{233}^{T} & Y_{223}^{T}+Y_{233}^{T} & \\
* & * & \hat{S}_{13}-\tilde{S}_{03}-\sum_{k=1}^{3} \tilde{R}_{03}^{k}-\tilde{R}_{13}^{2}-\tilde{R}_{13}^{3} & \tilde{R}_{13}^{2}+\tilde{R}_{13}^{3} & 0 & \\
* & * & * & \hat{S}_{23}-\tilde{S}_{13}-\tilde{R}_{23}^{1}-\tilde{R}_{13}^{2}-\tilde{R}_{13}^{3} & \tilde{R}_{23}^{1} & 0 \\
* & * & * & * & -\tilde{S}_{23}-\tilde{R}_{23}^{1} &
\end{array}\right],
$$$$
\Psi_{23}=\left[\begin{array}{cccccc}
Y_{13}^{T}-P_{23}^{T} \bar{A}_{13} & Y_{113}^{T} & Y_{123}^{T}-P_{23}^{T} \bar{A}_{23} & Y_{123}^{T} & Y_{133}^{T}-P_{23}^{T} \bar{A}_{33} & Y_{133}^{T} \\
Y_{213}^{T}-P_{33}^{T} \bar{A}_{13} & Y_{213}^{T} & Y_{223}^{T}-P_{33}^{T} \bar{A}_{23} & Y_{223}^{T} & Y_{233}^{T}-P_{33}^{T} \bar{A}_{33} & Y_{233}^{T} \\
0 & 0 & 0 & 0 & 0 & 0 \\
0 & 0 & 0 & 0 & 0 & 0 \\
0 & 0 & 0 & 0 & 0 & 0
\end{array}\right]
$$ 


$$
\begin{aligned}
& \Psi_{33}=\left[\begin{array}{cccccc}
-\tilde{R}_{13}^{1} & 0 & 0 & 0 & 0 & 0 \\
* & -\tilde{R}_{13}^{1} & 0 & 0 & 0 & 0 \\
* & * & -\tilde{R}_{23}^{2} & 0 & 0 & 0 \\
* & * & * & -\tilde{R}_{23}^{2} & 0 & 0 \\
* & * & * & * & -\tilde{R}_{23}^{3} & 0 \\
* & * & * & * & * & -\tilde{R}_{23}^{3}
\end{array}\right], \\
& \Psi_{14}=\left[\begin{array}{ccccc}
\Phi_{114} & \Phi_{124} & \sum_{k=1}^{3} \tilde{R}_{04}^{k}+P_{24}^{T} A_{34}-Y_{134}^{T} & P_{24}^{T} A_{14}+Y_{134}^{T}-Y_{114}^{T}-Y_{124}^{T} & Y_{114}^{T}+Y_{124}^{T} \\
* & \Phi_{224} & P_{34}^{T} A_{34}-Y_{234}^{T} & P_{34}^{T} A_{14}+Y_{234}^{T}-Y_{214}^{T}-Y_{224}^{T} & Y_{214}^{T}+Y_{224}^{T} \\
* & * & \hat{S}_{14}-\tilde{S}_{04}-\sum_{k=1}^{2}\left(\tilde{R}_{04}^{k}+\tilde{R}_{14}^{k}\right)-\tilde{R}_{04}^{3} & \sum_{k=1}^{2} \tilde{R}_{14}^{k} & 0 \\
* & * & * & \hat{S}_{24}-\tilde{S}_{14}-\sum_{k=1}^{2} \tilde{R}_{14}^{k}-\tilde{R}_{24}^{3} & \tilde{R}_{24}^{3} \\
* & * & * & * & -\tilde{S}_{24}-\tilde{R}_{24}^{3}
\end{array}\right] \text {, } \\
& \Psi_{24}=\left[\begin{array}{cccccc}
Y_{114}^{T}-P_{24}^{T} A_{14} & Y_{114}^{T} & Y_{12}^{T} & Y_{124}^{T} & Y_{13}^{T}-P_{24}^{T} A_{34} & Y_{134}^{T} \\
Y_{214}^{T}-P_{34}^{T} A_{14} & Y_{214}^{T} & Y_{224}^{T} & Y_{224}^{T} & Y_{234}^{T}-P_{34}^{T} A_{34} & Y_{234}^{T} \\
0 & 0 & 0 & 0 & 0 & 0 \\
0 & 0 & 0 & 0 & 0 & 0 \\
0 & 0 & 0 & 0 & 0 & 0
\end{array}\right] \\
& \Psi_{34}=\left[\begin{array}{cccccc}
-\tilde{R}_{24}^{1} & 0 & 0 & 0 & 0 & 0 \\
* & -\tilde{R}_{24}^{1} & 0 & 0 & 0 & 0 \\
* & * & -\tilde{R}_{24}^{2} & 0 & 0 & 0 \\
* & * & * & -\tilde{R}_{24}^{2} & 0 & 0 \\
* & * & * & * & -\tilde{R}_{14}^{3} & 0 \\
* & * & * & * & * & -\tilde{R}_{14}^{3}
\end{array}\right] \text {, }
\end{aligned}
$$

and where

$$
\begin{aligned}
& \tilde{R}_{i j}=e^{-2 \alpha_{j}\left(h_{i+1}\right)} R_{i j}, \\
& \tilde{S}_{i j}=e^{-2 \alpha_{j} h_{i+1}} S_{i j}, \\
& \hat{S}_{i j}=e^{-2 \alpha_{j} h_{i}} S_{i j}, \\
& \Phi_{11 j}=A^{T} P_{2 j}+P_{2 j}^{T} A+S_{0 j}-e^{-2 \alpha_{j} h_{1}} R_{0 j}+2 \alpha_{j} P_{j}, \\
& \Phi_{12 j}=P_{j}-P_{2 j}^{T}+A^{T} P_{3 j}, \Phi_{22 j}=-P_{3 j}-P_{3 j}^{T}+\sum_{i=0}^{2}\left(h_{i+1}-h_{i}\right)^{2} R_{i j} .
\end{aligned}
$$

Since the NCS global model switches are in a predetermined order, the global performance/stability is achieved if a one of the functional $V_{j}$ is decreasing each cycle. This gives the following condition to ensure a decay rate $\alpha_{g}$ over a complete cycle: $V_{1}\left(t_{s}, x_{t_{s}}, \dot{x}_{t_{s}}\right) \leq e^{-2 \alpha_{j}\left(t_{s}-t_{0}\right)} V_{1}\left(t_{0}, x_{t_{0}}, \dot{x}_{t_{0}}\right)$. This condition is illustrated on figure 3. The following theorem gives the stability conditions for a 4 sequenced mode switching system.

system (IV-A) has positive functionals (35) fulfilling 36. 
Theorem 2: Consider a 4 sequenced mode switching model described in (IV-A). Given some scalars $\alpha_{j}$ and $\mu_{i j},(i, j) \in\{(1,4),(4,2),(2,3),(3,1)\}$, if there exist scalars $\mu_{i j}, i, j=1, \ldots, 4$, matrices $P_{j}>0, R_{i j}^{k}>0$ and $S_{i j}>0(i=0,1,2$ and $k=1,2,3), P_{2 j}, P_{3 j}, Y_{1 k j}$ and $Y_{2 k j}(j=1,2,3,4$ and $k=1,2,3)$ with proper dimensions, such that (37), (38), (39) and condition (53) hold for some $\alpha_{g}>0$ then the model (IV-A) is exponentially stable with the decay rate $\alpha_{g}$ :

$$
\mu_{14} \mu_{42} \mu_{23} \mu_{31} e^{-2 \alpha_{4}\left(t_{s}-t_{3}\right)-2 \alpha_{2}\left(t_{3}-t_{2}\right)-2 \alpha_{3}\left(t_{2}-t_{1}\right)-2 \alpha_{1}\left(t_{1}-t_{0}\right)} \leq e^{\alpha_{g}\left(t_{s}-t_{0}\right)}
$$

Proof: Using the properties (36), it comes:

$$
\begin{aligned}
V_{1}\left(t_{s}, x_{t_{s}}, \dot{x}_{t_{s}}\right) & \leq \mu_{14} V_{4}\left(t_{s}, x_{t_{s}}, \dot{x}_{t_{s}}\right) \\
& \leq \mu_{14} e^{-2 \alpha_{4}\left(t_{s}-t_{3}\right)} V_{4}\left(t_{3}, x_{t_{3}}, \dot{x}_{t_{3}}\right) \\
& \leq \mu_{14} \mu_{42} e^{-2 \alpha_{4}\left(t_{s}-t_{3}\right)} V_{2}\left(t_{3}, x_{t_{3}}, \dot{x}_{t_{3}}\right) \\
& \leq \mu_{14} \mu_{42} e^{-2 \alpha_{2}\left(t_{s}-t_{2}\right)-2 \alpha_{2}\left(t_{3}-t_{2}\right)} V_{2}\left(t_{2}, x_{t_{2}}, \dot{x}_{t_{2}}\right) \\
& \cdots \\
& \leq \mu_{14} \mu_{42} \mu_{23} \mu_{31} e^{-2 \alpha_{4}\left(t_{s}-t_{3}\right)-2 \alpha_{2}\left(t_{3}-t_{2}\right)-2 \alpha_{3}\left(t_{2}-t_{1}\right)-2 \alpha_{1}\left(t_{1}-t_{0}\right)} V_{1}\left(t_{0}, x_{t_{0}}, \dot{x}_{t_{0}}\right) .
\end{aligned}
$$

then $V_{1}\left(t_{s}, x_{t_{s}}, \dot{x}_{t_{s}}\right) \leq e^{-2 \alpha_{j}\left(t_{s}-t_{0}\right)} V_{1}\left(t_{0}, x_{t_{0}}, \dot{x}_{t_{0}}\right)$ if

$$
\mu_{14} \mu_{42} \mu_{23} \mu_{31} e^{-2 \alpha_{4}\left(t_{s}-t_{3}\right)-2 \alpha_{2}\left(t_{3}-t_{2}\right)-2 \alpha_{3}\left(t_{2}-t_{1}\right)-2 \alpha_{1}\left(t_{1}-t_{0}\right)} \leq e^{\alpha_{g}\left(t_{s}-t_{0}\right)}
$$

Remark 5: There is always a solution to the problem of finding the switch time $t_{s}$ in the two following cases:

1) $\alpha_{g}<\alpha_{4}$ even in the case of fugitive modes,

2) $\alpha_{g}<\alpha_{1}$ if the dwell time in mode $S S_{1}$ is sufficient.

This result could be used to provide recommendation, in the form of dwell time in SS1 mode to choose the network type needed to achieved given performances.

Note that the conditions given in corollary 4 and in theorem 2 are not strictly LMI since the $\alpha_{j}$ and the $\mu_{i j}$ must be fixed. A successive optimization of the parameters can be achieved to get a compromise between a high values of $\alpha_{j}$ and low values $\mu_{i j}$. This optimization is possible since the conditions are harder to satisfy when $\alpha_{j}$ grows $/ \mu_{i j}$ decreases.

The global approach for the switching controller design is summarized in the following algorithm: 
Algorithm 1: Controller design steps:

1) use lemmas 1 and 2 to compute the gains with given $\alpha$ performances for modes 1 and 2 .

2) use corollary 4 to compute a compromise between the minimum $\mu_{i j}$ and the maximum $\alpha_{i}$.

3) implement the controller (7) that uses the following switching law:

$$
i=\left\{\begin{array}{l}
\left.1, \text { if } \delta_{o b s}(t)\right) \in S_{1} \text { and } t>t_{s} \\
2, \text { otherwise. }
\end{array}\right.
$$

$t_{s}$ is extracted by applying the logarithm function on the condition of theorem 2 .

Remark 6: These result does not take into account the packet dropout case for sake of clarity. Since the presented controller does not detect packet dropouts, it is treated using [30] to compute de worst case exponential decay rate for all modes. Remark also that these results can be extended to more zones and to the case where the control delay range is different from the observer delay range.

\section{EXPERIMENTAL RESULTS}

The experiments are done on two computers separated from about 40 kilometers away (Fig. 4). The Master program runs on the remote computer with an advanced computing capability, the Slave one on the local one which also communicates with a light-inertia robot Miabot of the company Merlin by Bluetooth.

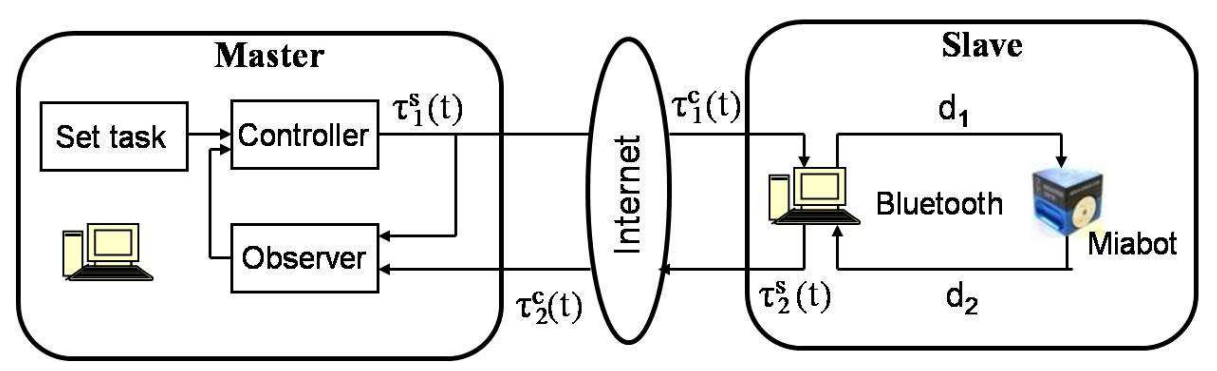

Fig. 4. Structure of the global system

\section{A. The structure of the Master}

In order to implement the model for the remote control system, a four-threads program is designed to fulfill the functions of Controller and Observer in Fig.4, while the explication of all the parameters refers to [15]. 


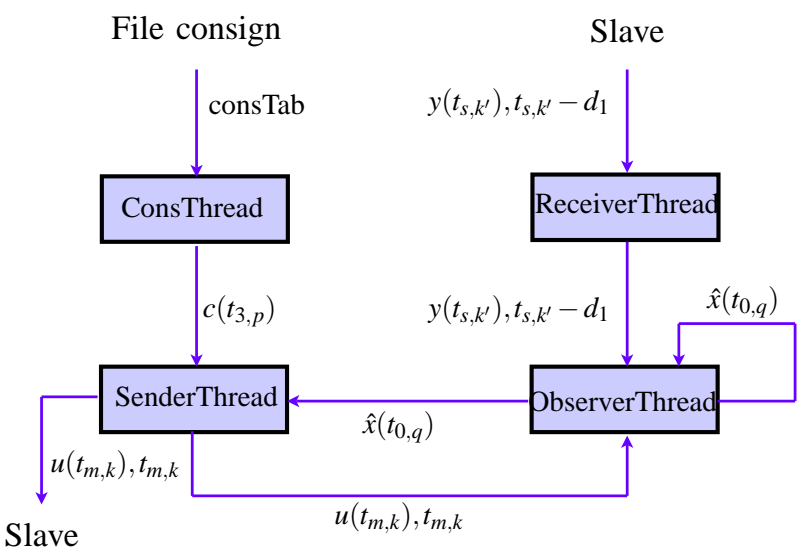

Fig. 5. Structure of the Master

These four threads are concurrently working as showed in Fig.5. (a) ConsThread is a periodic thread which gets the set points of the trajectory. (b) SenderThread is also a periodic thread which calculates the control data and its appliance time and send it out to the Slave. (c) ReceiverThread is a event-driven thread to receive controls from the Slave. (d) ObserverThread task is periodic and simulate the observers differential equations. It estimates the present position and speed of the remote motor.

\section{B. The structure of the Slave}

The Slave does not need power computation abilities, because it just needs to communicate with the Master and the Miabot. As we can see from Fig.6, this program is divided into two threads: ReceiveThread and SendThread. (a) ReceiveThread is an event-driven thread which is activated by the control data arrived from the Master. (b) SendThread is used to apply the control to the Miabot as well as to get its real position, and then send the data back to the Master. As we need to apply the control data at the time given by the master, the buffer used is a list list_Y where the packet are sorted by order of appliance.

\section{Experimental study}

The model of the Miabot is derived experimentally. In order to avoid saturation and nonlinear behaviors, the speed has been limited to $2 \mathrm{~m} / \mathrm{sec}$. The obtained model is given by (57). The state value is composed of its position and speed, the control is the value sent by Master. 


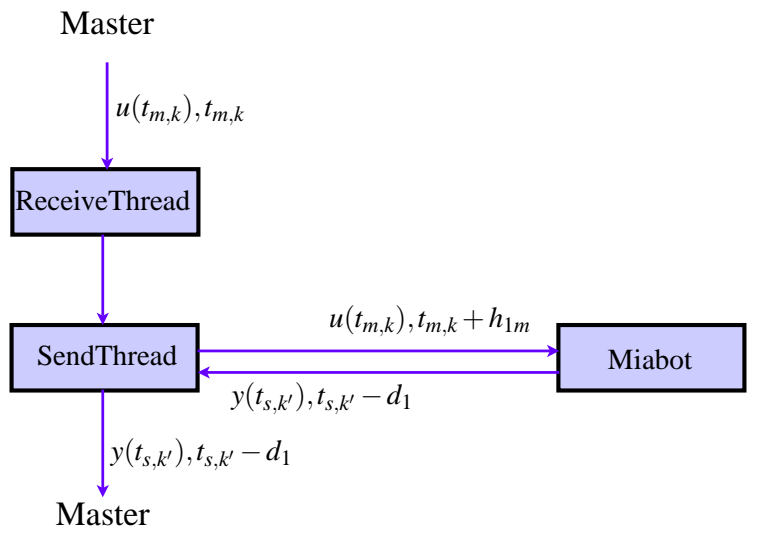

Fig. 6. Structure of the Slave

$$
\left\{\begin{array}{l}
\dot{x}(t)=\left[\begin{array}{cc}
0 & 1 \\
0 & -10
\end{array}\right] x(t)+\left[\begin{array}{c}
0 \\
0.024
\end{array}\right] u\left(t-\delta_{\text {con }}(t)\right) \\
y(t)=\left[\begin{array}{ll}
1 & 0
\end{array}\right] x(t)
\end{array}\right.
$$

Some delay measurement has been done between the master and the slave. During a day, the RTT (Round-trip-time=twice the communication delay) measured using the ICMP (Internet Control Message [25]) belonged to $[4.1 \mathrm{~ms} 577 \mathrm{~ms}]$ with an average value of $52.37 \mathrm{~ms}$. During the experimental time, the delay was oscillating between an average value of $40 \mathrm{~ms}$ and $100 \mathrm{~ms}$. Taking into account these information, considering also the Bluetooth transmission delays (considered constant) and the sampling delays, we take $S_{1}=\left[0.01,0.08\left[\right.\right.$ and $S_{2}=[0.08,0.5]$ for the delay subset. Any packet data delayed by more than $0.5 \mathrm{sec}$ is considered lost.

According to Lemma (1) and (2), the maximum exponential convergence ensuring the modes stability are: $\alpha_{1}^{c}=3.8, \alpha_{1}^{o}=4.49, \alpha_{2}^{c}=\alpha_{2}^{o}=0.72$. The corresponding control gains are too high to keep the speed lower than $2 \mathrm{~m} / \mathrm{sec}$. To avoid actuator saturation and nonlinear behavior when the robot speed is too high, smaller gains are computed considering the following values : $\alpha_{1}^{c}=2, \alpha_{1}^{o}=2.5, \alpha_{2}^{c}=0.6$ and $\alpha_{2}^{o}=0.72$.

The resulting gains $K_{i}$ and $L_{i}(i=1,2)$ are given by:

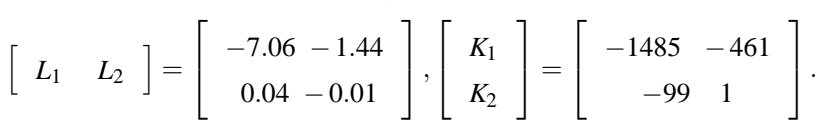

Remark 7: Note that speed related component of the control gain $K_{2}$ has a positive value. This just mean that to keep the system stability when the delay is high, it is needed slightly degrade the performances of the system stable open-loop pole. 
Once the gain are computed, the dwell time parameters $\alpha_{i}$ and $\mu_{i j}$ are computed following theses steps:

1) The values of $\alpha_{3}$ and $\alpha_{4}$ are maximized for fixed $\mu_{i j}=100, \alpha_{1}$ and $\alpha_{2}$, satisfying corollary 4 conditions.

2) $\mu_{i j}$ are minimized using the criterion $\mu_{14}+\mu_{42}+\mu_{23}+\mu_{31}$ for the obtained $\alpha_{i}$ and LKF matrices, satisfying corollary 4 conditions.

These steps lead to the following solutions: $\alpha_{1}=2, \alpha_{2}=0.6, \alpha_{3}=-4, \alpha_{4}=0.6$. The global performance $\alpha_{g}$ chosen is chosen to only keep stability: $\alpha_{g}=0$.

\section{Results of remote experiment}

The result is shown in Fig.7, in which the blue curve represents the set values; the green and red represent respectively the robot's estimated position and speed; the black corresponds to the real position of the Miabot. Fig.8 illustrate the corresponding switched control signals from Master to Slave. The red curve is the real control while the green and the black ones are the controls calculated respectively for the two subsystems. We can see the switch points according to the values of time-delay. Fig.9 depicts the variable global time-delays on the control communication channel and the corresponding switching signal.

On Fig.7, one can notice three kinds of step responses. The first one corresponds to the case when the time-delay is greater than $80 \mathrm{~ms}$, only the second subsystem is active. In this case, a decay rate $\alpha_{2}$ is guaranteed. During the second step, only the first mode is active because delays are small. The performances are better: the response time is smaller since a decay rate of $\alpha_{1}$ is achieved. In the last kind of response, where a some switches occur during the transient response. In that case, only the global stability is guaranteed. At the moment $t \in[42 \mathrm{~ms} 45 \mathrm{~ms}]$, the time-delay becomes small, but the switching strategy () do not permit to switch back to mode 1 in order to guarantee the global stability.

Remark 8: Notice that despite the fact that the observer is based on delays measurements, it this keep a good estimation on the present state of the slave.

The last figure (Fig.10) provides a performance analysis in term of global decay rate $\alpha_{g}$ over one cycle $\left(S S_{1} \rightarrow S U_{1} \rightarrow S S_{2} \rightarrow S U_{2} \rightarrow S S_{1}\right)$. It is assumed that the dwell time in the modes $S U 1$ and $S U 2$ are fixed to the maximum delay, i.e. $0.5 s$. The figure gives the lower bound $\alpha_{g}$ according the the dwell time $T$ in the modes $S S 1$ and $S S 2$. For $T \leq 3.7$ the controller dwell 


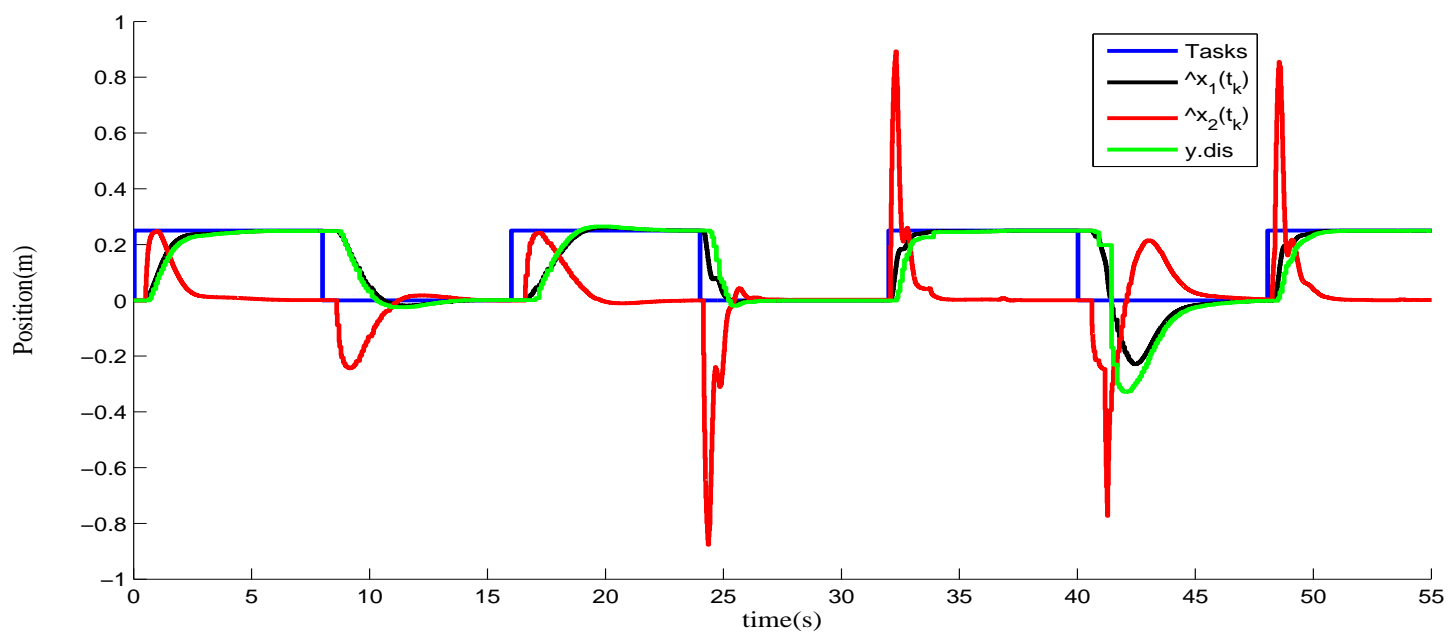

Fig. 7. Results of remote experiment

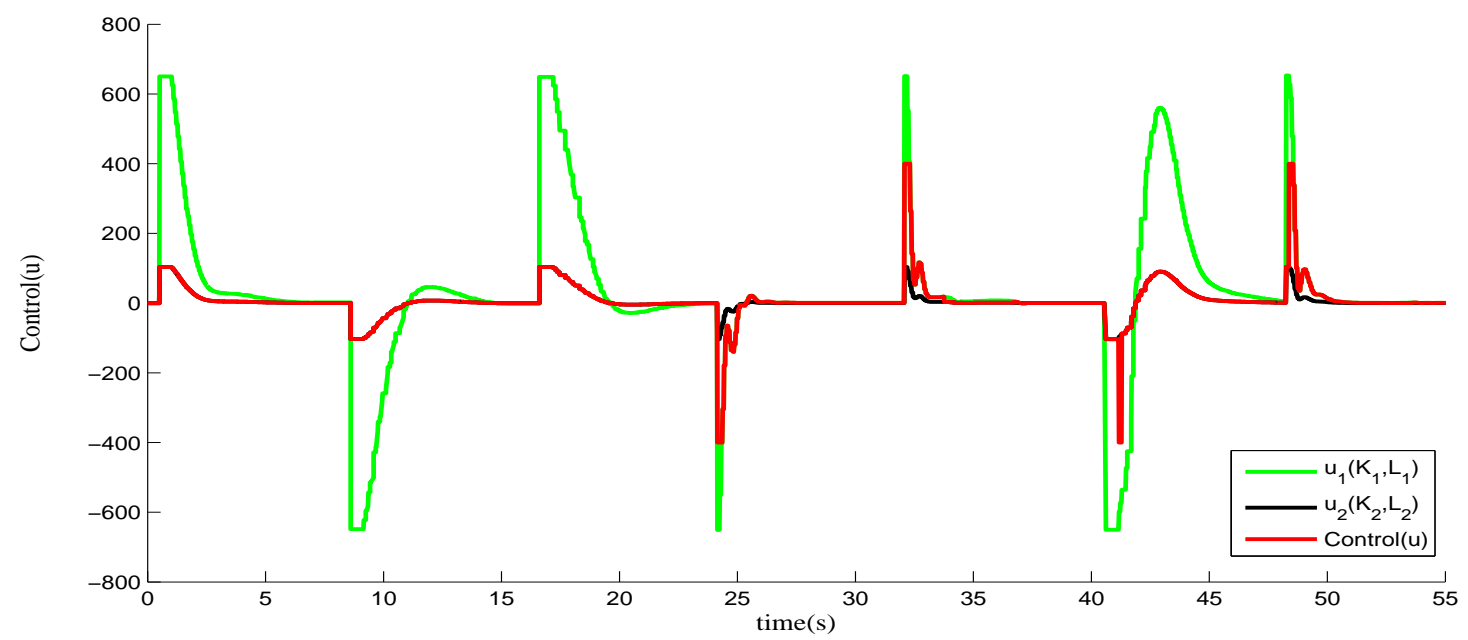

Fig. 8. The corresponding switched control

July 23, 2010

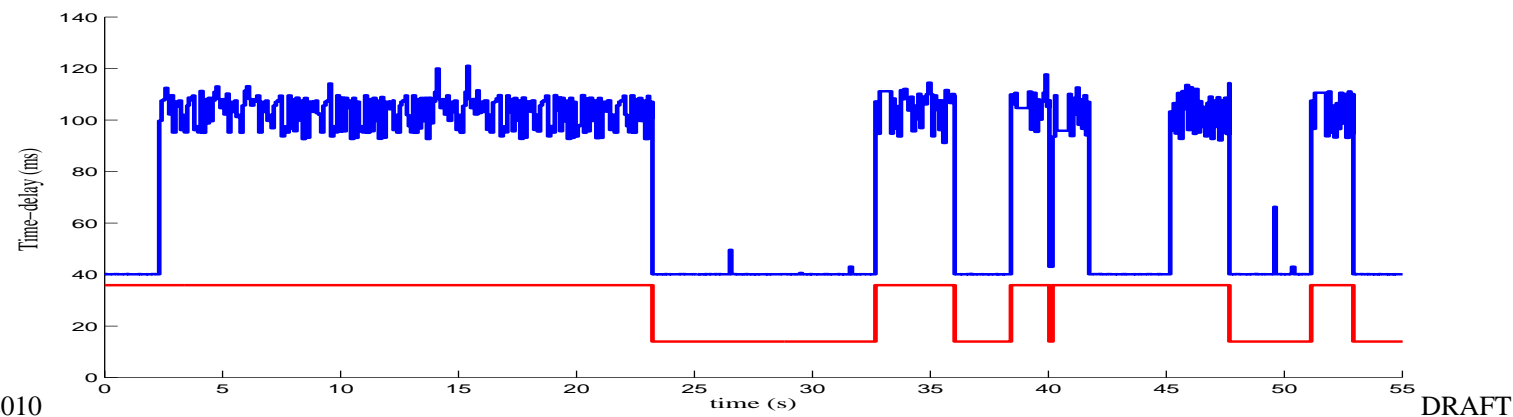

Fig. 9. The corresponding variable time-delays and signals of switching 


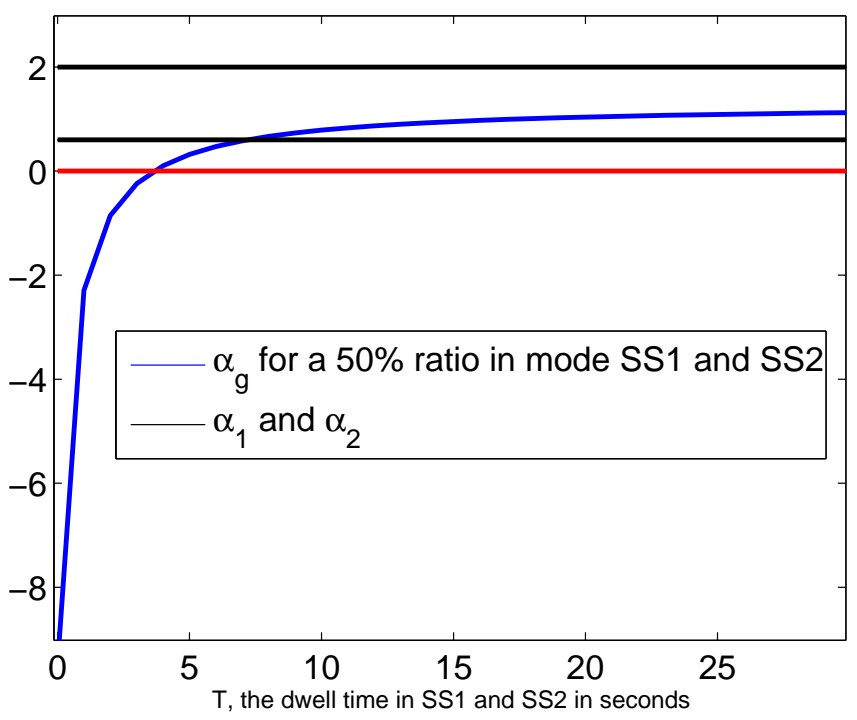

Fig. 10. Performances analysis

time condition is not satisfied which leads to a possible unstable behavior. For $T \in[3.7$ 7.2] the performances are worse than $\alpha_{2}=0.6$ and for $T>7.2$, the performances tends to the mean values of the decays rates $\left(\alpha_{1}+\alpha_{2}\right) / 2=1.3$.

\section{CONCLUSION}

A QoS-dependent controller has been presented to enhance the performances of a NCS system based on switching gains. This controller uses the time-stamps of packets to measure the communication delay and to synchronize the observer with the plant. The resulting controller switches according to the delay and a dwell time strategy ensuring the global stability.

For the sake of clarity, only two intervals for the delay range are considered to quantify the QoS but the conditions can be easily extended to the multiple intervals.

An experimental platform has been presented and illustrates the results of the network-based control theory, showing the effectiveness of the dwell time strategy.

\section{REFERENCES}

[1] Chen Z., Liu L., and Yin X., "Networked control system with network time-delay compensation," Industry Applications Conference, Fourtieth IAS Annual Meeting, vol. 4, pp. 2435 - 2440, 102005. 
[2] Chiasson J. and Loiseau J.J., Applications of time delay systems. Springer, 2007, vol. 352.

[3] Estrada-Garcia H.J., Marquez-Martinez L.A., and Moog C.H., "Master-slave synchronization for two inverted pendulums with communication time-delay," 7th IFAC workshop on time delay systems, Nantes, France, 2007.

[4] Fattouh A. and Sename O., " $\mathrm{H}^{\infty}$-based impedance control of teleoperation systems with time delay," 4th Workshop on Time Delay Systems, 92003.

[5] Fridman E., "New Lyapunov-Krasovskii functionals for stability of linear retarded and neutral type systems," Systems \& Control Letters, vol. 43, pp. 309-319, 2001.

[6] Fridman E., Seuret A., and Richard J.-P., "Robust sampled-data stabilization of linear systems: an input delay approach," Automatica, vol. 40, pp. 1441-1446, 2004.

[7] Gao H., Chen T., and Lam J., "A new delay system approach to network-based control," Automatica, vol. 44, no. 1, pp. 39-52, 2008.

[8] Georges J.-P., Divoux T., and Rondeau E., "Confronting the performances of a switched ethernet network with industrial constraints by using the network calculus," International Journal of Communication Systems(IJCS), vol. 18, no. 9, pp. 877-903, 2005.

[9] Gu K., Kharitonov V., and Chen J., "Stability of time-delay systems," Birkhauser: Boston, 2003.

[10] He Y., Wu M., She J.H., and Liu G.P., "Parameter-dependent lyapunov functional for stability of time-delay systems with polytopic-type uncertainties," IEEE Transactions on Automatic Control, vol. 49, pp. 828-832, 2004.

[11] Hespanha J.P. and Morse A.S., "stability of switched systems with average dwell-time," Proceedings of the $38^{\text {th }}$ Conference on Decision \& Control, pp. 2655-2660, December 1999.

[12] Hespanha J.P., Naghshtabrizi P., and Xu Y., "A survey of recent results in networked control systems," Proceedings of the IEEE, vol. 95, pp. 138-162, January 2007.

[13] Hirche S., Chen C.-C, and Buss M, "Performance oriented control over networks -switching controllers and switched time delay-," Proceedings of the 45th IEEE Conference on Decision \& Control, December 2006.

[14] Huang J.Q. and Lewis F.L., "Neural-network predictive control for nonlinear dynamic systems with time delays," IEEE Transactions on Neural Networks, vol. 14, no. 2, pp. 377-389, 2003.

[15] Jiang W.-J., Kruszewski A., Richard J.-P., and Toguyeni A., "A gain scheduling strategy for the control and estimation of a remote robot via internet," The 27th Chinese Control Conference, July 2008.

[16] Lelevé A., Fraisse P., and Dauchez P., “Telerobotics over IP networks: Towards a low-level real-time architecture,” IROS'01 International conference on intelligent robots and systems,Maui,Hawaii, October 2001.

[17] Liberzon D., Switching in Systems and Control, T. Basar, Ed. Birkhäuser, 2003.

[18] Mills D.L., "Improved algorithms for synchronizing computer network clocks," IEEE/ACM Transactions On Networking, vol. 3, no. 3, pp. 245-254, June 1995.

[19] Niculescu S.-I., Delay effects on stability: a robust control approach. Springer, 2001, vol. 269.

[20] Niemeyer G. and Slotine J.-J., “Towards force-reflecting teleoperation over the internet," IEEE Int. Con. on Robotics \& Automation, 1998.

[21] Nilsson J., "Real-time control systems with delays," Ph.d. thesis at the department of automatic control. Lund Institute of Technology. Sweden, 1998.

[22] Nilsson J., Bernhardsson B., and Wittenmark B., "Stochastic analysis and control of real-time control systems with random time delay." Automatica, vol. 34, no. 1, pp. 57-64, 1998. 
[23] Ploplys N.J., Kawka P.A., and Alleyne A.G., "Closed-loop control over wireless networks," IEEE Control Systems Magazine, June 2004.

[24] J. Postel, "User datagram protocol," USC/Information Sciences Institute, RFC 768, 1980.

[25] — - "Internet control message protocol," USC/Information Sciences Institute, RFC 792, 1981.

[26] Richard J.-P., "Time delay systems: an overview of some recent advances and open problems," Automatica, vol. 39, pp. 1667-1694, 2003.

[27] Richard J.-P. and Divoux T., Systèmes commandés en réseau. Hermes-Lavoisier, IC2, Systèmes Automatisés, 2007.

[28] Seuret A., Fridman E., and Richard J.-P., "Sampled-data exponential stabilization of neutral systems with input and state delays," Proc. of IEEE MED 2005, 13th Mediterranean Conference on Control and Automation, Cyprus, 2005.

[29] Seuret A., Michaut F., Richard J.-P., and Divoux T., "Networked control using gps synchronization," Proc. of ACC06, American Control Conf., Mineapolis, USA, June 2006.

[30] Seuret A. and Richard J.-P., "Control of a remote system over network including delays and packet dropout," IFAC World Congress, Seoul, Korea, July 2008.

[31] Sun X.-M., Zhao J., and Hill D., "Stability and $l_{2}$-gain analysis for switched delay systems," Automatica, vol. 42, pp. 1769-1774, 2006.

[32] Sun Y.G. and Wang L., "Stability of switched systems with time-varying delays: delay-dependent common lyapunov functional approach," Proceedings of the 2006 American Control Conference, June 2006.

[33] Tipsuwan Y. and Chow M.-Y., "Control methodologies in network control systems," Control Engineering Practice, vol. 11, pp. 1099-1011, 2003.

[34] Witrant E., Canudas-De-Wit C., and Georges D., "Remote stabilization via communication networks with a distributed control law," IEEE Transactions on Automatic control, 2007.

[35] Yan P. and Özbay H., "Stability analysis of switched time delay systems," SIAM Journal on Control and Optimization, vol. 47, no. 2, pp. 936-949, 2008.

[36] Yu M., Wang L., and Chu T., "An LMI approach to network control systems with data packet dropout and transmission delays," MTNS '04 Proc. of Mathematical Theory Networks and Systems, Leuven, Belgium, 2004.

[37] Zampieri S., “Trends in networked control systems," Proceedings of the 17th World Congress, The International federation of Automatic Control, July 2008.

[38] Zhang W.A. and Yu L., "Modelling and control of networked control systems with both network-induced delay and packet-dropout," Automatica, vol. 44, pp. 3206-3210, 2008. 